\title{
A Combined Strain Element in Static, Frequency and Buckling Analyses of Laminated Composite Plates and Shells
}

\author{
Hoang Lan Ton-That ${ }^{1,2^{*}}$, Hieu Nguyen-Van ${ }^{2}$ \\ ${ }^{1}$ Faculty of Civil Engineering, Ho Chi Minh City University of Technology and Education, 01 Vo Van Ngan Street, Thu Duc District, Ho Chi \\ Minh City, Vietnam \\ 2 Faculty of Civil Engineering, Ho Chi Minh City University of Architecture,196 Pasteur Street, District 3, Ho Chi Minh City, Vietnam \\ * Corresponding author, e-mail: lan.tonthathoang@uah.edu.vn
}

Received: 08 July 2020, Accepted: 02 September 2020, Published online: 01 October 2020

\begin{abstract}
This paper deals with numerical analyses of laminated composite plate and shell structures using a new four-node quadrilateral flat shell element, namely SQ4C, based on the first-order shear deformation theory (FSDT) and a combined strain strategy. The main notion of the combined strain strategy is based on the combination of the membrane strain and shear strain related to tying points as well as bending strain with respect to cell-based smoothed finite element method. Many desirable characteristics and the enforcement of the SQ4C element are verified and proved through various numerical examples in static, frequency and buckling analyses of laminated composite plate and shell structures. Numerical results and comparison with other reference solutions suggest that the present element is accuracy, efficiency and removal of shear and membrane locking.
\end{abstract}

Keywords

flat shell element, laminated composite plate and shell, first-order shear deformation theory (FSDT), shear locking, membrane locking

\section{Introduction}

Laminated composite materials are manufactured by stacking layers of orthotropic materials in different orientations to obtain the desired strength and stiffness. Nowadays, the laminated composite materials are frequently applied in many industries because of their attractive properties as compared to isotropic materials, such as higher stiffness-to-weight ratio, strength, damping and good properties related to thermal or acoustic isolation, among others. Due to the vast application of composite materials in the industries, extensive research has been carried out to achieve a better understanding of the behaviors of composite structures. A fast growing interest in the use of laminated composite plate and shell structures in engineering field is demonstrated by various efforts to expand the robust analysis. There are many theories introduced into linear and nonlinear analyses from thin to thick plates or shells such as the classical plate theory (CPT), the first-order shear deformation theory (FSDT), the higher-order shear deformation theory (HSDT), the layer-wise theory (LWT) and variable kinematics models. Amongst them, the first-order shear deformation theory (FSDT) is commonly used because of its low computational cost and simplicity. And besides, numerical methods have been expanded for the analysis of laminated composite plate and shell structures as given by Yang et al. [1], Ko etal. [2], Ton-That et al. [3] and so on.

Specifically, we can mention a survey of recent shell finite elements which includes the degenerated shell approach, stress-resultant-based formulations and Cosserat surface approach, reduced integration with stabilization, incompatible modes approach, enhanced strain formulations, 3-D elasticity elements, drilling degree of freedom elements, co-rotational approach and higher-order theories for composites. Besides the standard finite element methods, the smoothed finite element formulation for static, free vibration and buckling analyses of laminated composite plates and shells was also introduced in [4]. The improved four-node elements for analysis of composite plate/shell structures based on twice interpolation strategy was given by Ton-That et al. [5]. Many desirable characteristics of these efficient numerical methods were shown as continuous nodal gradients, higher order polynomial basis, no increase in number of the degree of freedom of the system. In [6], a novel numerical procedure based on the 
framework of isogeometric analysis was presented for static, free vibration, and buckling analysis of laminated composite plates using the first-order shear deformation theory. The isogeometric approach utilizes non-uniform rational B-splines to implement for the quadratic, cubic, and quartic elements. Shear locking problem could be significantly alleviated by a stabilization technique. In our research, a new 4-node quadrilateral flat shell element, namely SQ4C, is introduced for linear analysis of the laminated composite plate and shell structures. Although the 4-node shell finite elements MITC4 are popular because of their accuracy and simplicity, the MITC4 elements usually endure the membrane locking when curved geometries of the structures are solved with distorted meshes. Ko et al. [2] have suggested novel assumed membrane strain fields, which are simple and effective for reducing the membrane locking. The authors call the MITC4+ shell elements. However, both MITC4 and MITC4+ elements do not employ any smoothing techniques for the bending strains. This can cause some troubles of convergence rate when using badly distorted meshes. Therefore, the present SQ4C flat shell element is proposed by using the strain smoothing technique for the bending strains combined with the assumed membrane strains as well as shear strains through tying points. The SQ4C can reduce affection of shear and membrane locking. Numerical examples indicate that the present element is free from locking and reveals good stability as well as accuracy in linear analysis, including statics, frequency and buckling of laminated composite plates and shells.

This paper is summarized as follows. A terse review of the first-order shear deformation theory (FSDT) is firstly introduced in Section 2. The formulation of the SQ4C flat shell element is presented in Section 3. Various numerical examples are fulfilled in Section 4. Some conclusions are finally given in Section 5.

\section{A brief of the first-order shear deformation theory (FSDT)}

A laminated composite plate is considered as shown in Fig. 1. It has a thickness $h$ with $n$ orthotropic layers. The $k^{\text {th }}$ layer is situated between the line $z=z_{k-1}$ and $z=z_{k}$, moreover, the $x y$-plane is called the undeformed mid-surface of the laminate. The first-order shear deformation theory (FSDT) of laminated composite plates is an enhancement of the Reissner-Mindlin theory [7]. The kinematics of the plate is determined by the mid-surface displacements $u_{0}, v_{0}, w_{0}$ and rotations $\theta_{x}, \theta_{y}$ as follows:

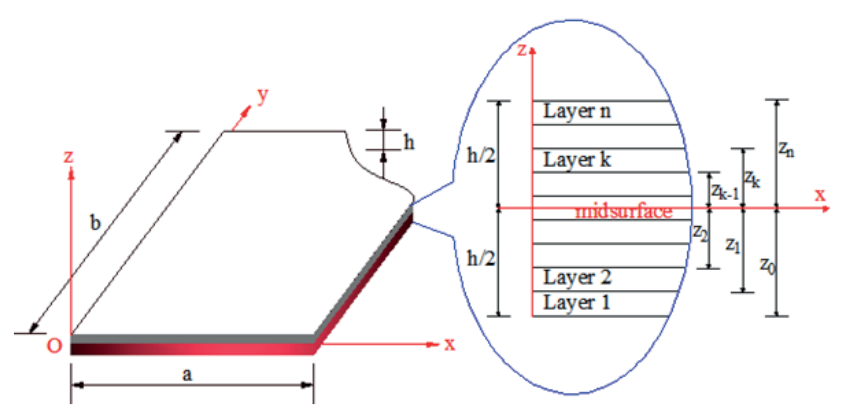

Fig. 1 The laminated composite plate with thickness $h$ and $n$ orthotropic layers

$u(x, y, z)=u_{0}(x, y)+z \theta_{y}$

$v(x, y, z)=v_{0}(x, y)-z \theta_{x}$,

$w(x, y, z)=w_{0}(x, y)$,

in which $u_{0}, v_{0}, w_{0}$ are called the displacements of a point located in the mid-surface, and $\theta_{x}, \theta_{y}$ are called the rotations of the transverse normal, i.e. in the $z$ direction, about the $x$ - as well as $y$-axes, respectively.

The in-plane strain vector can be rewritten

$\boldsymbol{\varepsilon}=\left[\begin{array}{c}\varepsilon_{x} \\ \varepsilon_{y} \\ \gamma_{x y}\end{array}\right]=\underbrace{\left[\begin{array}{c}u_{0, x} \\ v_{0, y} \\ u_{0, y}+v_{0, x}\end{array}\right]}_{\varepsilon^{m}}+z \underbrace{\left[\begin{array}{c}\theta_{y, x} \\ -\theta_{x, y} \\ \theta_{y, y}-\theta_{x, x}\end{array}\right]}_{\varepsilon^{b}}=\varepsilon^{m}+z \varepsilon^{b}$,

and the transverse shear strain vector is also presented

$\varepsilon^{s}=\left[\begin{array}{c}\varepsilon_{x z} \\ \varepsilon_{y z}\end{array}\right]=\left[\begin{array}{c}\theta_{y}+w_{0, x} \\ -\theta_{x}+w_{0, y}\end{array}\right]$.

The relationships between the stresses and strains are described in matrix notations as below:

$\sigma_{p}=\left[\begin{array}{l}\overline{\boldsymbol{N}} \\ \overline{\boldsymbol{M}}\end{array}\right]=\left[\begin{array}{ll}\overline{\boldsymbol{A}} & \overline{\boldsymbol{B}} \\ \overline{\boldsymbol{B}} & \overline{\boldsymbol{D}}\end{array}\right]\left[\begin{array}{l}\varepsilon^{m} \\ \boldsymbol{\varepsilon}^{b}\end{array}\right]$,

$\overline{\boldsymbol{S}}=\left[\begin{array}{cc}k_{1}^{2} \bar{C}_{55}^{0} & k_{1} k_{2} \bar{C}_{45}^{0} \\ k_{1} k_{2} \bar{C}_{45}^{0} & k_{2}^{2} \bar{C}_{44}^{0}\end{array}\right]\left[\begin{array}{l}\varepsilon_{x z} \\ \varepsilon_{y z}\end{array}\right]=\overline{\boldsymbol{C}}_{s} \varepsilon^{s}$.

with $\overline{\boldsymbol{N}}=\left[N_{x} N_{y} N_{x y}\right]^{T}, \overline{\boldsymbol{M}}=\left[M_{x} M_{y} M_{x y}\right]^{T}$, and $\overline{\boldsymbol{S}}=\left[Q_{x} Q_{y}\right]$ are called the membrane force vector, the bending moment vector and the transverse shear force vector; $k_{1}^{2}, k_{2}^{2}$ are the shear correction factors (SCFs); $\overline{\boldsymbol{A}}, \overline{\boldsymbol{B}}, \overline{\boldsymbol{D}}$ and $\overline{\boldsymbol{C}}$ are called the matrices of extensional stiffness, bending-extension coupling stiffness, bending stiffness and transverse shearing stiffness, succinctly depicted as

$\left(\bar{A}_{i j}, \bar{B}_{i j}, \bar{D}_{i j}\right)=\int_{-h / 2}^{h / 2}\left(1, z, z^{2}\right) \bar{Q}_{i j} d z, \quad i, j=1,2,6$. 


$$
\bar{C}_{i j}^{0}=\int_{-h / 2}^{h / 2} \bar{Q}_{i j} d z, \quad i, j=4,5 .
$$

In which $\bar{Q}_{i j}$ are called the elastic constants in the $x$-axis with their details were obtained in [7].

\section{A novel 4-node quadrilateral flat shell element SQ4C for laminated composite structures}

3.1 The standard formulation of the 4-node quadrilateral flat shell element in the local coordinate system

Consider a plate or shell structure discretized by 4-node quadrilateral flat shell elements. A local coordinate system $O x y z$ is defined for each element, in which the $O x y$ plane is the mid-surface of the element. The displacement approximations of the 4-node quadrilateral flat shell element are defined in the local coordinate system as follows

$$
\begin{aligned}
& u_{0}=\sum_{i=1}^{4} N_{i}(\xi, \eta) u_{i} ; v_{0}=\sum_{i=1}^{4} N_{i}(\xi, \eta) v_{i} ; \\
& w_{0}=\sum_{i=1}^{4} N_{i}(\xi, \eta) w_{i} ; \\
& \theta_{x}=\sum_{i=1}^{4} N_{i}(\xi, \eta) \theta_{x i} ; \theta_{y}=\sum_{i=1}^{4} N_{i}(\xi, \eta) \theta_{y i} .
\end{aligned}
$$

In which $N_{i}(\xi, \eta)=0.25\left(1+\xi_{i} \xi\right)\left(1+\eta_{i} \eta\right)$ denotes the shape function of the element; $\left(\xi_{i}, \eta_{i}\right)$ is the nodal coordinates in the nature coordinate system $(\xi, \eta)$; and $u_{i}, v_{i}, w_{i}$, $q_{x i}, q_{y i}$ are the nodal displacements of the element with the positive directions given in Fig. 2.

From Eq. (2), the discrete strain field based on the derivatives of the displacement approximation (Eq. (8)) can be given

$$
\begin{aligned}
& \boldsymbol{\varepsilon}^{m}=\sum_{i=1}^{4} \boldsymbol{B}_{m i} \boldsymbol{q}_{m i}=\underbrace{\left[\begin{array}{llll}
\boldsymbol{B}_{m 1} & \boldsymbol{B}_{m 2} & \boldsymbol{B}_{m 3} & \boldsymbol{B}_{m 4}
\end{array}\right]}_{\mathbf{B}_{m}} \boldsymbol{q}_{m}=\boldsymbol{B}_{m} \boldsymbol{q}_{m}, \\
& \boldsymbol{\varepsilon}^{b}=\sum_{i=1}^{4} \boldsymbol{B}_{b i} \boldsymbol{q}_{b i}=\underbrace{\left[\begin{array}{llll}
\boldsymbol{B}_{b 1} & \boldsymbol{B}_{b 2} & \boldsymbol{B}_{b 3} & \boldsymbol{B}_{b 4}
\end{array}\right]}_{\mathbf{B}_{b}} \boldsymbol{q}_{b}=\boldsymbol{B}_{b} \boldsymbol{q}_{b},
\end{aligned}
$$

with, $\boldsymbol{q}_{m i}=\left[\begin{array}{ll}u_{i} & v_{i}\end{array}\right]^{T}, \boldsymbol{q}_{b i}=\left[w_{i} \theta_{x i} \theta_{y i}\right]^{T}$ and the gradient matrices

$$
\boldsymbol{B}_{m i}=\left[\begin{array}{cc}
N_{i, x} & 0 \\
0 & N_{i, y} \\
N_{i, y} & N_{i, x}
\end{array}\right] ; \quad \boldsymbol{B}_{b i}=\left[\begin{array}{ccc}
0 & 0 & N_{i, x} \\
0 & -N_{i, y} & 0 \\
0 & -N_{i, x} & N_{i, y}
\end{array}\right] .
$$

Similarly, the relationship between the transverse shear strain and nodal displacements can be written

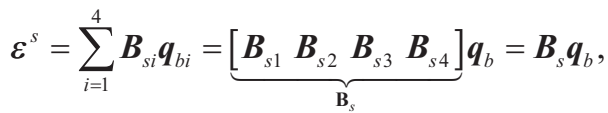
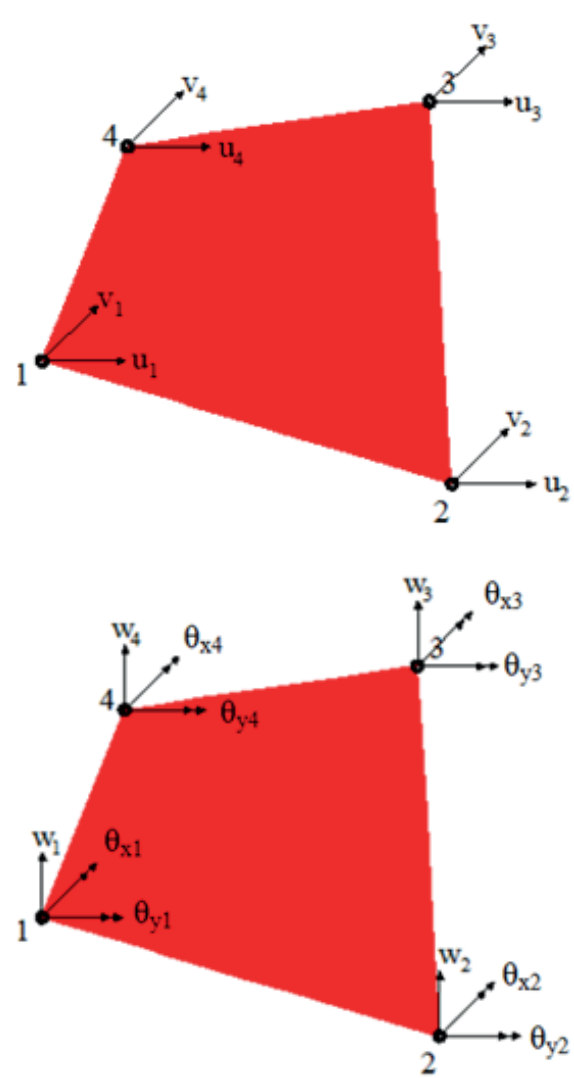

Fig. 2 Nodal displacements of a 4-node quadrilateral element

with

$$
\boldsymbol{B}_{s i}=\left[\begin{array}{ccc}
N_{i, x} & 0 & N_{i} \\
N_{i, y} & -N_{i} & 0
\end{array}\right] .
$$

According to the standard finite element procedure, the formulas of the element stiffness matrix $\boldsymbol{K}_{e}$, the element force vector $\boldsymbol{F}_{e}$, the mass matrix $\boldsymbol{M}_{e}$ and the geometric stiffness matric $\boldsymbol{K}_{e}^{g}$ in the local coordinate system can be respectively obtained

$$
\boldsymbol{K}_{e}=\left[\begin{array}{ccc}
\boldsymbol{K}_{e}^{m} & \boldsymbol{K}_{e}^{m b} & \mathbf{0} \\
\left(\boldsymbol{K}_{e}^{m b}\right)^{T} & \boldsymbol{K}_{e}^{b} & \mathbf{0} \\
\mathbf{0} & \mathbf{0} & k_{z}
\end{array}\right]
$$

$\boldsymbol{F}_{e}=\int_{\Omega_{e}} \boldsymbol{N}^{T} \boldsymbol{p} \mathrm{d} \Omega$

$\boldsymbol{M}_{e}=\left[\begin{array}{cc}\int_{\Omega_{e}} \boldsymbol{N}_{m}^{T} \boldsymbol{m} \boldsymbol{N}_{m} \mathrm{~d} \Omega & \mathbf{0} \\ \mathbf{0} & m_{z}\end{array}\right]$,

$\boldsymbol{K}_{e}^{g}=\int_{\Omega_{e}} \boldsymbol{B}_{g}^{T} \hat{\sigma}_{0} \boldsymbol{B}_{g} \mathrm{~d} \Omega$ 
In which

$$
\begin{aligned}
& \boldsymbol{K}_{e}^{m}=\int_{\Omega_{e}} \boldsymbol{B}_{m}^{T} \boldsymbol{A} \boldsymbol{B}_{m} \mathrm{~d} \Omega \\
& \boldsymbol{K}_{e}^{b}=\int_{\Omega_{e}} \boldsymbol{B}_{b}^{T} \boldsymbol{D} \boldsymbol{B}_{b} \mathrm{~d} \Omega+\int_{\Omega_{e}} \boldsymbol{B}_{s}^{T} \boldsymbol{C}_{s} \boldsymbol{B}_{s} \mathrm{~d} \Omega ; \\
& \boldsymbol{K}_{e}^{m b}=\int_{\Omega_{e}} \boldsymbol{B}_{m}^{T} \boldsymbol{B} \boldsymbol{B}_{b} \mathrm{~d} \Omega
\end{aligned}
$$$$
\boldsymbol{m}=\rho h\left[\begin{array}{ccccc}
1 & 0 & 0 & 0 & 0 \\
0 & 1 & 0 & 0 & 0 \\
0 & 0 & 1 & 0 & 0 \\
0 & 0 & 0 & \frac{h^{2}}{12} & 0 \\
0 & 0 & 0 & 0 & \frac{h^{2}}{12}
\end{array}\right]
$$

$N, N_{m}$ are the matrices of the shape functions; $\boldsymbol{p}$ is the vector of external loadings; and $\rho$ is the mass density.

$\boldsymbol{B}_{g}=\left[\begin{array}{llll}\boldsymbol{B}_{g 1} & \boldsymbol{B}_{g 2} & \boldsymbol{B}_{g 2} & \boldsymbol{B}_{g 2}\end{array}\right]$ with

$\boldsymbol{B}_{g i}=\left[\begin{array}{ccccc}N_{i, x} & 0 & 0 & 0 & 0 \\ N_{i, y} & 0 & 0 & 0 & 0 \\ 0 & N_{i, x} & 0 & 0 & 0 \\ 0 & N_{i, y} & 0 & 0 & 0 \\ 0 & 0 & N_{i, x} & 0 & 0 \\ 0 & 0 & N_{i, y} & 0 & 0 \\ 0 & 0 & 0 & N_{i, x} & 0 \\ 0 & 0 & 0 & N_{i, y} & 0 \\ 0 & 0 & 0 & 0 & N_{i, x} \\ 0 & 0 & 0 & 0 & N_{i, y}\end{array}\right]$,

$\hat{\sigma}_{0}=\left[\begin{array}{ccccc}h \sigma_{0} & \mathbf{0} & \mathbf{0} & \mathbf{0} & \mathbf{0} \\ \mathbf{0} & h \boldsymbol{\sigma}_{0} & \mathbf{0} & \mathbf{0} & \mathbf{0} \\ \mathbf{0} & \mathbf{0} & h \sigma_{0} & \mathbf{0} & \mathbf{0} \\ \mathbf{0} & \mathbf{0} & v & \frac{h^{3}}{12} \sigma_{0} & \mathbf{0} \\ \mathbf{0} & \mathbf{0} & \mathbf{0} & \mathbf{0} & \frac{h^{3}}{12} \sigma_{0}\end{array}\right]$ with

$\sigma_{0}=\left[\begin{array}{cc}\sigma_{x}^{0} & \sigma_{x y}^{0} \\ \sigma_{x y}^{0} & \sigma_{y}^{0}\end{array}\right]$.

And $k_{z}=10^{-6} \times \max \left(\operatorname{diag}\left(\boldsymbol{K}_{e}\right)\right)$ and $m_{z}=10^{-6} \times \max (\mathrm{di}-$ $\left.\operatorname{ag}\left(\boldsymbol{M}_{e}\right)\right)$ are factors used to eliminate the singularity due to the artificial drilling degree-of-freedom.

\subsection{A novel 4-node quadrilateral flat shell element SQ4C}

\subsubsection{Membrane part}

The mid-surface of this element is subdivided into four non-overlapping 3-node triangular domains defined by the vertexes and the center point ' 5 ' of the element as shown in Fig. 3. The coordinates of the point ' 5 ' in the natural coordinate system are interpolated by [2].

$$
\boldsymbol{x}_{5}=\sum_{i=1}^{4} \varsigma_{i} \boldsymbol{x}_{i}
$$

Here, the constants $\varsigma_{i}$ are determined using the following equation:

$$
\begin{aligned}
{\left[\begin{array}{llll}
\varsigma_{1} & \varsigma_{2} & \varsigma_{3} & \varsigma_{4}
\end{array}\right] } & =\frac{1}{2} \frac{A_{234}}{A_{234}+A_{124}}\left[\begin{array}{llll}
\frac{1}{3} & \frac{1}{3} & 0 & \frac{1}{3}
\end{array}\right] \\
& +\frac{1}{2} \frac{A_{124}}{A_{234}+A_{124}}\left[\begin{array}{llll}
0 & \frac{1}{3} & \frac{1}{3} & \frac{1}{3}
\end{array}\right] \\
& +\frac{1}{2} \frac{A_{134}}{A_{134}+A_{123}}\left[\begin{array}{llll}
\frac{1}{3} & \frac{1}{3} & \frac{1}{3} & 0
\end{array}\right] \\
& +\frac{1}{2} \frac{A_{123}}{A_{134}+A_{123}}\left[\begin{array}{llll}
\frac{1}{3} & 0 & \frac{1}{3} & \frac{1}{3}
\end{array}\right] .
\end{aligned}
$$

In which, $A_{234}, A_{124}, A_{134}$ and $A_{123}$ are the areas of triangles '234', '124', '134' and '123' defined in Fig. 4. Based on the characteristics of the isoparametric element, the in-plane displacement vector of the point ' 5 ' is approximated from the nodal displacements as

$\boldsymbol{q}_{m 5}=\sum_{i=1}^{4} \varsigma_{i} \boldsymbol{q}_{m i}$.

To alleviate the membrane locking, Ko et al. [2] have approximated the membrane strains as

$$
\begin{aligned}
& \tilde{\varepsilon}^{m}=\frac{1}{4}\left(\tilde{\varepsilon}^{m(A)}+\tilde{\varepsilon}^{m(B)}+\tilde{\varepsilon}^{m(C)}+\tilde{\varepsilon}^{m(D)}\right) \\
& +\frac{1}{2}\left(-\tilde{\varepsilon}^{m(D)}+\tilde{\varepsilon}^{m(C)}\right) \xi+\frac{1}{2}\left(-\tilde{\varepsilon}^{m(B)}+\tilde{\varepsilon}^{m(A)}\right) \eta,
\end{aligned}
$$

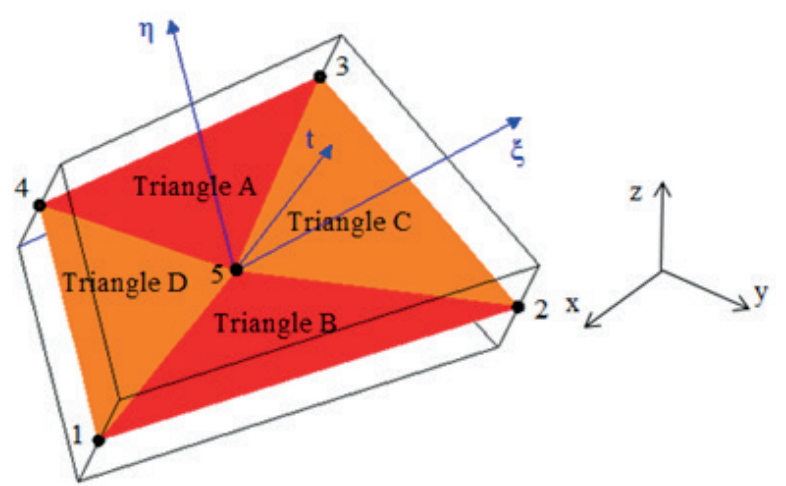

Fig. 3 Triangular subdivision of the mid-surface of the 4-node element 
In which, and are respectively the membrane strains of the 3-node triangular domains A, B, C and D evaluated at the tying points (A), (B), (C) and (D) with their positions in the natural coordinate system given in Fig. 5.

From the displacement approximation, and relationships between the membrane strains and nodal displacements, the assumed membrane strains can be described

$\tilde{\boldsymbol{\varepsilon}}^{m}=\tilde{\boldsymbol{B}}_{m} \boldsymbol{q}_{m}$.
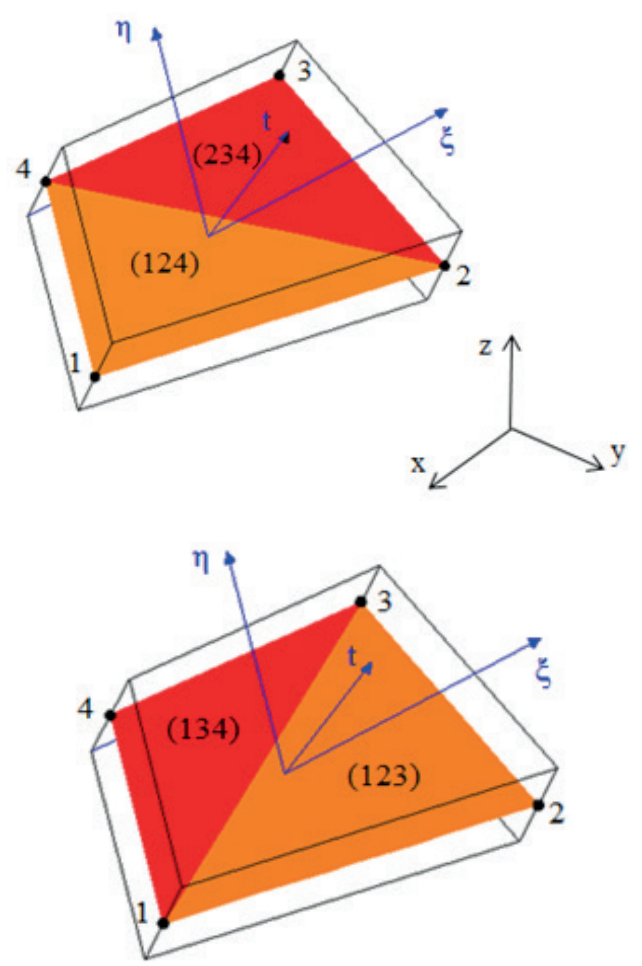

Fig. 4 Four triangles to determine the point ' 5 ' of the element

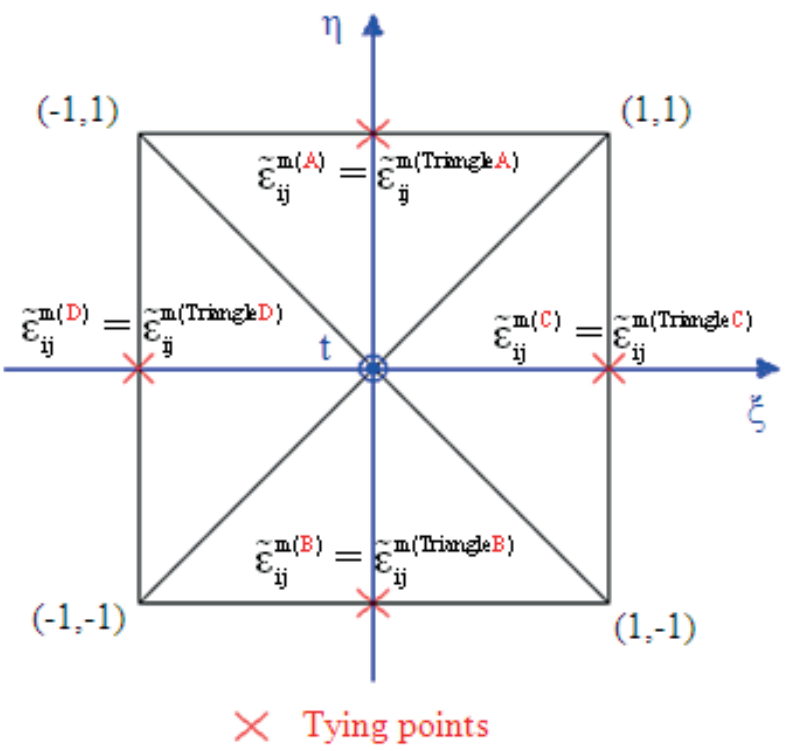

Fig. 5 Position of tying points (A), (B), (C) and (D) corresponding to the four non-overlapping triangular domains

\subsubsection{Bending part}

The bending strain will be smoothed by following [4] and as shown in Fig. 6. Thence the liaison between the nodal displacements and the smoothed bending strain field is rewritten as

$\tilde{\boldsymbol{\varepsilon}}^{b}=\sum_{i=1}^{n C} \tilde{\boldsymbol{B}}_{b i} \boldsymbol{q}_{b i}=\tilde{\boldsymbol{B}}_{b} \boldsymbol{q}_{b}$,

where

$\tilde{\boldsymbol{B}}_{b i}=\frac{1}{A_{C}} \int_{\Gamma_{C}}\left[\begin{array}{ccc}0 & N_{i} n_{x} & 0 \\ 0 & 0 & N_{i} n_{y} \\ 0 & N_{i} n_{y} & N_{i} n_{x}\end{array}\right] \mathrm{d} \Gamma$.

Here, $A_{c}$ and $\Gamma_{c}$ are respectively the area and the boundary of the smoothing cell. $n_{x}$ and $n_{y}$ are the components of the vector normal to the boundary $\Gamma_{c}$.

\subsubsection{Shear part}

The transverse shear strain field is based on assuming constant transverse shear strain conditions along the edges to attenuate the shear locking phenomenon [8] as follows

$\tilde{\varepsilon}_{\xi t}^{s}=\frac{1}{2}(1+\eta) \varepsilon_{\xi t}^{s(E)}+\frac{1}{2}(1-\eta) \varepsilon_{\xi t}^{s(F)}$,
$\tilde{\varepsilon}_{\eta t}^{s}=\frac{1}{2}(1+\xi) \varepsilon_{\eta t}^{s(G)}+\frac{1}{2}(1-\xi) \varepsilon_{\eta t}^{s(H)}$.
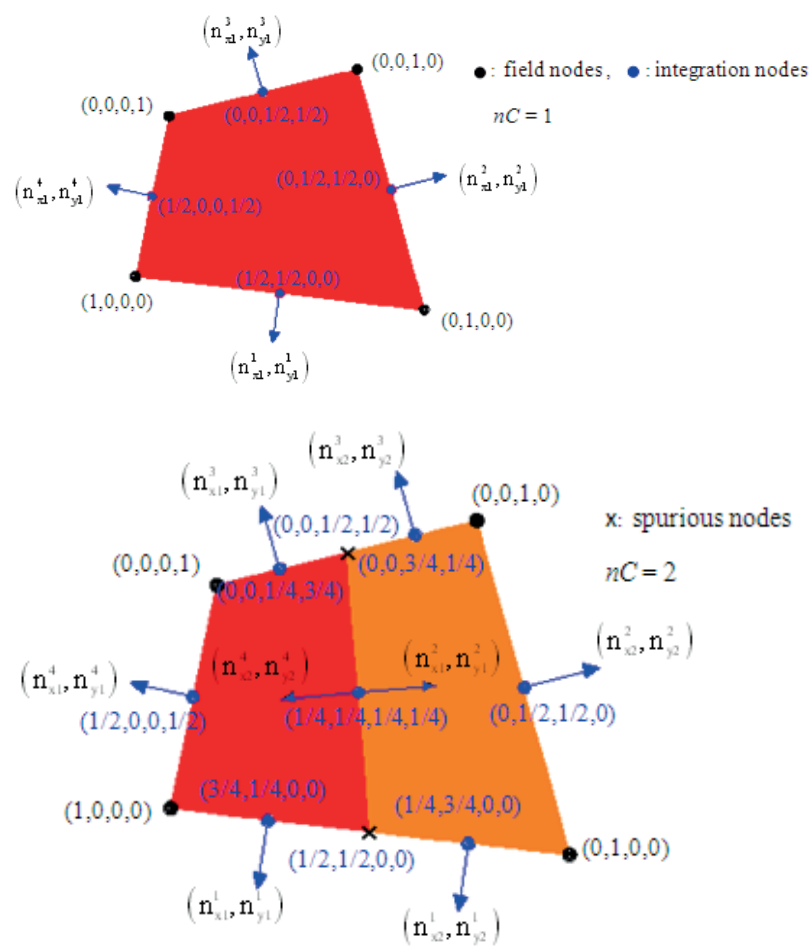

Fig. 6 Subdivision of the 4-node quadrilateral element into $n C$ smoothing cells and the set of shape functions at nodes in the format $\left(N_{1}, N_{2}, N_{3}, N_{4}\right)$ 
Here, and are the transverse shear strains directly computed from the displacement approximation at the tying points shown in Fig. 7.

$\tilde{\boldsymbol{B}}_{s i}=\left[\begin{array}{ll}x_{, \xi} & y_{, \xi} \\ x_{, \eta} & y_{, \eta}\end{array}\right]^{-1}\left[\begin{array}{lll}\mathbf{N}_{i, \xi} & b_{i}^{11} \mathbf{N}_{i, \xi} & b_{i}^{12} \mathbf{N}_{i, \xi} \\ \mathbf{N}_{i, \eta} & b_{i}^{21} \mathbf{N}_{i, \eta} & b_{i}^{22} \mathbf{N}_{i, \eta}\end{array}\right]$,

where $b_{i}^{11}=\xi_{i} x_{\xi}^{M}, b_{i}^{12}=\xi_{i} y_{\xi}^{M}, b_{i}^{21}=\eta_{i} x_{\eta}^{L}$ and $b_{i}^{22}=\eta_{i} y_{\eta}^{L}$ in which $\xi_{i} \in\left\{\begin{array}{llll}-1 & 1 & 1 & -1\end{array}\right\}, \eta_{i} \in\left\{\begin{array}{llll}-1 & -1 & 1 & 1\end{array}\right\}$ and $(i, M, L) \in\{(1, F, H),(2, F, G)(3, E, G),(4, E, H)\}$

\subsubsection{The combined strain strategy}

As a result, the stiffness matrix and the geometric stiffness matrix of the 4-node quadrilateral flat shell element SQ4C in the local coordinate system are rewritten

$\tilde{\boldsymbol{K}}_{e}=\left[\begin{array}{ccc}\tilde{\boldsymbol{K}}_{e}^{m} & \tilde{\boldsymbol{K}}_{e}^{m b} & \mathbf{0} \\ \left(\tilde{\boldsymbol{K}}_{e}^{m b}\right)^{T} & \tilde{\boldsymbol{K}}_{e}^{b} & \mathbf{0} \\ \mathbf{0} & \mathbf{0} & k_{z}\end{array}\right]$,

$\tilde{\boldsymbol{K}}_{e}^{g}=\int_{\Omega_{e}} \tilde{\boldsymbol{B}}_{g}^{T} \widehat{\boldsymbol{\sigma}}_{0} \tilde{\boldsymbol{B}}_{g} \mathrm{~d} \Omega$,

here,

$$
\begin{aligned}
& \tilde{\boldsymbol{K}}_{e}^{m}=\int_{\Omega_{e}} \tilde{\boldsymbol{B}}_{m}^{T} \boldsymbol{A} \tilde{\boldsymbol{B}}_{m} \mathrm{~d} \Omega, \\
& \tilde{\boldsymbol{K}}_{e}^{b}=\int_{\Omega_{e}} \tilde{\boldsymbol{B}}_{b}^{T} \boldsymbol{D} \tilde{\boldsymbol{B}}_{b} \mathrm{~d} \Omega+\int_{\Omega_{e}} \tilde{\boldsymbol{B}}_{s}^{T} \boldsymbol{C}_{s} \tilde{\boldsymbol{B}}_{s} \mathrm{~d} \Omega, \\
& \tilde{\boldsymbol{K}}_{e}^{m b}=\int_{\Omega_{e}} \tilde{\boldsymbol{B}}_{m}^{T} \boldsymbol{B} \tilde{\boldsymbol{B}}_{b} \mathrm{~d} \Omega,
\end{aligned}
$$

$\tilde{\boldsymbol{B}}_{g}=\left[\begin{array}{llll}\tilde{\boldsymbol{B}}_{g 1} & \tilde{\boldsymbol{B}}_{g 2} & \tilde{\boldsymbol{B}}_{g 3} & \tilde{\boldsymbol{B}}_{g 4}\end{array}\right]$ with

$$
\tilde{\boldsymbol{B}}_{g i}=\frac{1}{A_{C}} \int_{\Gamma_{C}}\left[\begin{array}{ccccc}
N_{i} n_{x} & 0 & 0 & 0 & 0 \\
N_{i} n_{y} & 0 & 0 & 0 & 0 \\
0 & N_{i} n_{x} & 0 & 0 & 0 \\
0 & N_{i} n_{y} & 0 & 0 & 0 \\
0 & 0 & N_{i} n_{x} & 0 & 0 \\
0 & 0 & N_{i} n_{y} & 0 & 0 \\
0 & 0 & 0 & N_{i} n_{x} & 0 \\
0 & 0 & 0 & N_{i} n_{y} & 0 \\
0 & 0 & 0 & 0 & N_{i} n_{x} \\
0 & 0 & 0 & 0 & N_{i} n_{y}
\end{array}\right] \mathrm{d} \Gamma \text {. }
$$

To build the structure stiffness and mass matrices, the element stiffness and mass matrices are transformed from the local coordinate system to the global coordinate system by
$\tilde{\boldsymbol{K}}_{e}^{\mathrm{glb}}=\boldsymbol{T}^{T} \tilde{\boldsymbol{K}}_{e} \boldsymbol{T}$,

$\mathbf{M}_{e}^{\mathrm{glb}}=\boldsymbol{T}^{T} \mathbf{M}_{e} \boldsymbol{T}$,

$\tilde{\boldsymbol{K}}_{e}^{g, g \mathrm{glb}}=\boldsymbol{T}^{T} \tilde{\boldsymbol{K}}_{e}^{g} \boldsymbol{T}$,

$\boldsymbol{T}=\left[\begin{array}{llll}\lambda & \mathbf{0} & \mathbf{0} & \mathbf{0} \\ \mathbf{0} & \lambda & \mathbf{0} & \mathbf{0} \\ \mathbf{0} & \mathbf{0} & \lambda & \mathbf{0} \\ \mathbf{0} & \mathbf{0} & \mathbf{0} & \lambda\end{array}\right]$.

In which, $\lambda$ is the direction cosine matrix transforming from the local coordinate system to the global coordinate system.

Finally, the problems of static, natural frequency and buckling analyses are solved by the following discretized equations:

$K q=F$,

$\left(\boldsymbol{K}-\omega^{2} \boldsymbol{M}\right) \boldsymbol{q}=\mathbf{0}$,

$\left(\boldsymbol{K}-\lambda \boldsymbol{K}_{g}\right) \boldsymbol{q}=\mathbf{0}$.

Here, $\boldsymbol{K}, \boldsymbol{M}$ and $\boldsymbol{K}_{g}$ are respectively the stiffness, mas and geometric stiffness matrices of the structures, which are assembled from the stiffness, mass and geometric stiffness matrices of the elements and $\tilde{\boldsymbol{K}}_{e}^{\mathrm{glb}}, \boldsymbol{M}_{e}^{\mathrm{glb}}$ and $\tilde{\boldsymbol{K}}_{e}^{g, \mathrm{glb}} ; \boldsymbol{q}$ is the nodal displacements of the structure; $\omega$ is the natural frequency; and $\lambda$ is the critical buckling load factor.

\section{Numerical examples}

In this section, the patch tests, the Cook's problem as well as various application studies are given to verify the recommendation of the SQ4C element in analyses of

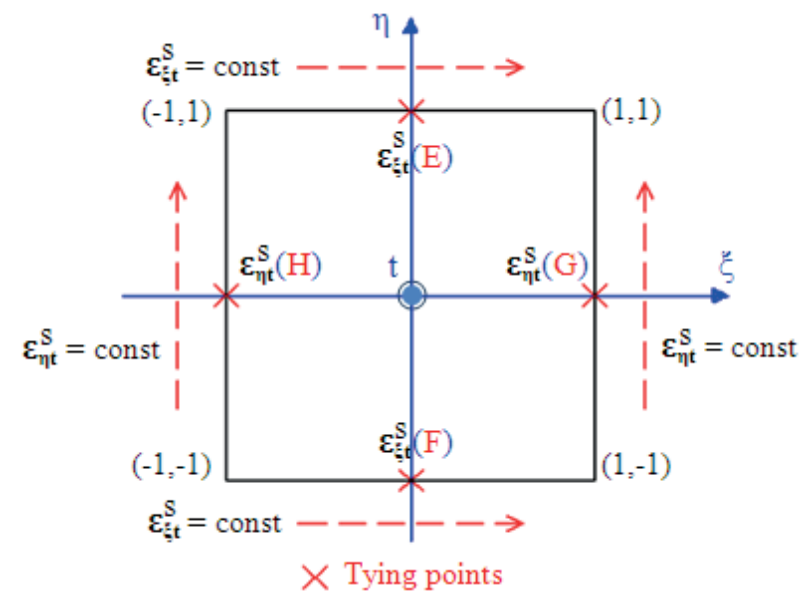

Fig. 7 Tying positions $(E),(F),(G)$ and $(H)$ for the assumed transverse shear strains 
several shapes of structures. The material properties for the laminated composite structures are accepted to be the equivalent in all the layers. From the global $x$-axis to the fiber direction, the ply angle is valuated for each layer. Moreover, based on the identification of the thickness of each layer, the units of the model are also assumed to be consistent and thence are not specified for almost examples. The SCFs are $k_{1}{ }^{2}=k_{2}{ }^{2}=5 / 6$ except for examples mentioned. The following Table 1 is three sets of material properties that are taken into the analyses.

\subsection{Basic tests}

\subsubsection{The patch test}

The patch tests are sufficient requirements in considering the convergence or the assignment of a novel element. We verify whether the stabilized SQ4C elements can reproduce a constant distribution of all quantities for distorted meshes. The material, the mesh as well as the boundary conditions were arrogated by using the details of [9] as depicted in Fig. 8. In these verifications, the nominated displacements are located at the edges related to four nodes $1,2,3$, and 4 . They are clearly given including in-plane patch test and out-of-plane patch test for membrane and bending situations.

Table 1 Three sets of material properties

\begin{tabular}{c} 
Material I \\
\hline$E_{1} / E_{2}=3,10,20,30,40 ; G_{12}=G_{13}=0.6 E_{2} ; G_{23}=0.5 E_{2} ;$ \\
$v_{12}=v_{13}=v_{23}=0.25, \rho=1$. \\
Material II \\
$E_{1} / E_{2}=25 ; G_{12}=G_{13}=0.5 E_{2} ; G_{23}=0.2 E_{2} ; v_{12}=v_{13}=v_{23}=0.25, \rho=1$. \\
Material III \\
$E_{1}=2.0685 \times 10^{11} ; E_{1} / E_{2}=40 ; G_{12}=G_{13}=0.5 E_{2} ; G_{23}=0.6 E_{2} ;$ \\
$v_{12}=v_{13}=v_{23}=0.25 ; \rho=1605$.
\end{tabular}
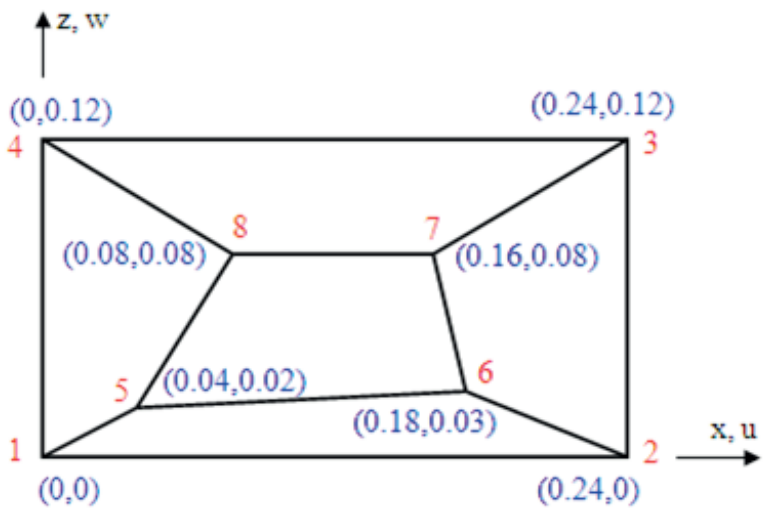

Fig. 8 The geometry and the mesh for patch test with material properties as $E=10^{6}, v=0.25, h=0.001$
The boundary conditions are taken into two verifications which related to the membrane patch test: $u=10^{-3}(x+0.5 y)$, $v=10^{-3}(y+0.5 x), w=0$ and the bending patch test: $w=10^{-3}\left(x_{2}+x y+y_{2}\right) / 2, \theta_{x}=10^{-3}(y+0.5 x), \theta_{y}=10^{-3}(x+0.5 y)$, $w=0$ The proposed element with exact results that up to 8 digit machine precision is proved through both patch tests as given in Table 2 .

\subsubsection{The Cook's problem}

With rerespect to test the in-plane bending and shearing behaviors of the SQ4C element, the Cook's problem shown in Fig. 9 is presented $[2,10]$.

The displacement at point $A$ in vertical direction as well as displacement field or stress field are given by using the SQ4C element as Table 3 and Fig. 10. This test shows that these behaviors are better than the behaviors of the MITC4 and MITC4+ shell elements respectively.

\subsection{Static analysis}

A fully clamped square plate with two-layer $\left[\theta^{\circ}-\theta^{\circ}\right]$, material $I$, length $a=10$ as well as thickness $h=0.02$ and subjected to a uniform load $q_{o}=1$ as shown in Fig. 11(a) is analyzed. The thickness of each layer is $h / 2$. Table 4 and Fig. 12(a) give a comparison of the normalized central deflection $\bar{w}=100 E_{2} w h^{3} /\left(q_{o} a^{4}\right)$ for this structure with

Table 2 The results of patch tests

\begin{tabular}{cccc}
\hline Test & Stress & SQ4C & Exact \\
\hline \multirow{3}{*}{ Membrane } & $\sigma_{x}$ & $1.33300000 \times 10^{3}$ & 1333 \\
& $\sigma_{y}$ & $1.33300000 \times 10^{3}$ & 1333 \\
& $\sigma_{x y}$ & $0.40000000 \times 10^{3}$ & 400 \\
& $M_{x}$ & $1.11111111 \times 10^{-7}$ & $1.11111111 \times 10^{-7}$ \\
& $M_{y}$ & $1.11111111 \times 10^{-7}$ & $1.11111111 \times 10^{-7}$ \\
& $M_{x y}$ & $0.33333333 \times 10^{-7}$ & $0.33333333 \times 10^{-7}$ \\
\hline
\end{tabular}
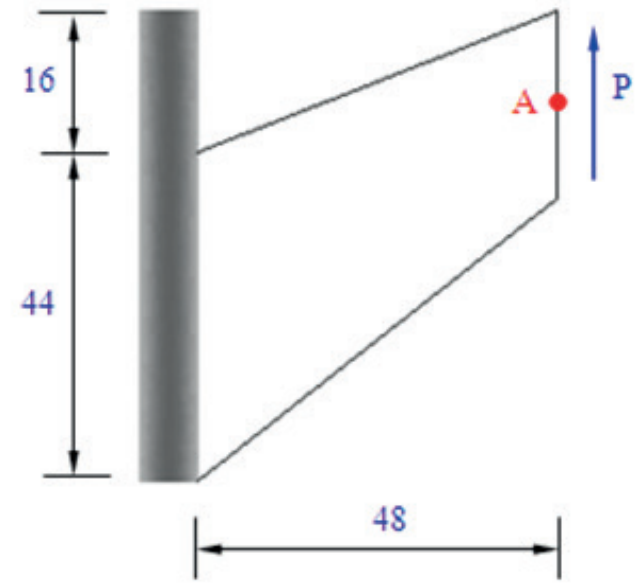

Fig. 9 The Cook's problem with 
Table 3 The vertical displacement at point A for Cook's problem

\begin{tabular}{lcccccc}
\hline Element & \multicolumn{5}{c}{ Mesh } \\
& $2 \times 2$ & $4 \times 4$ & $8 \times 8$ & $16 \times 16$ & $32 \times 32$ & Exact \\
\hline MITC4 & 11.8452 & 18.2992 & 22.0792 & 23.4304 & 23.8176 & 23.9642 \\
MITC4+ & 11.7291 & 18.2662 & 22.0751 & 23.4301 & 23.8176 & \\
SQ4C & $\mathbf{1 2 . 2 5 7 6}$ & $\mathbf{1 8 . 5 1 4 9}$ & $\mathbf{2 2 . 1 5 1 3}$ & $\mathbf{2 3 . 4 5 0 7}$ & $\mathbf{2 3 . 8 2 3 0}$ & \\
\hline
\end{tabular}
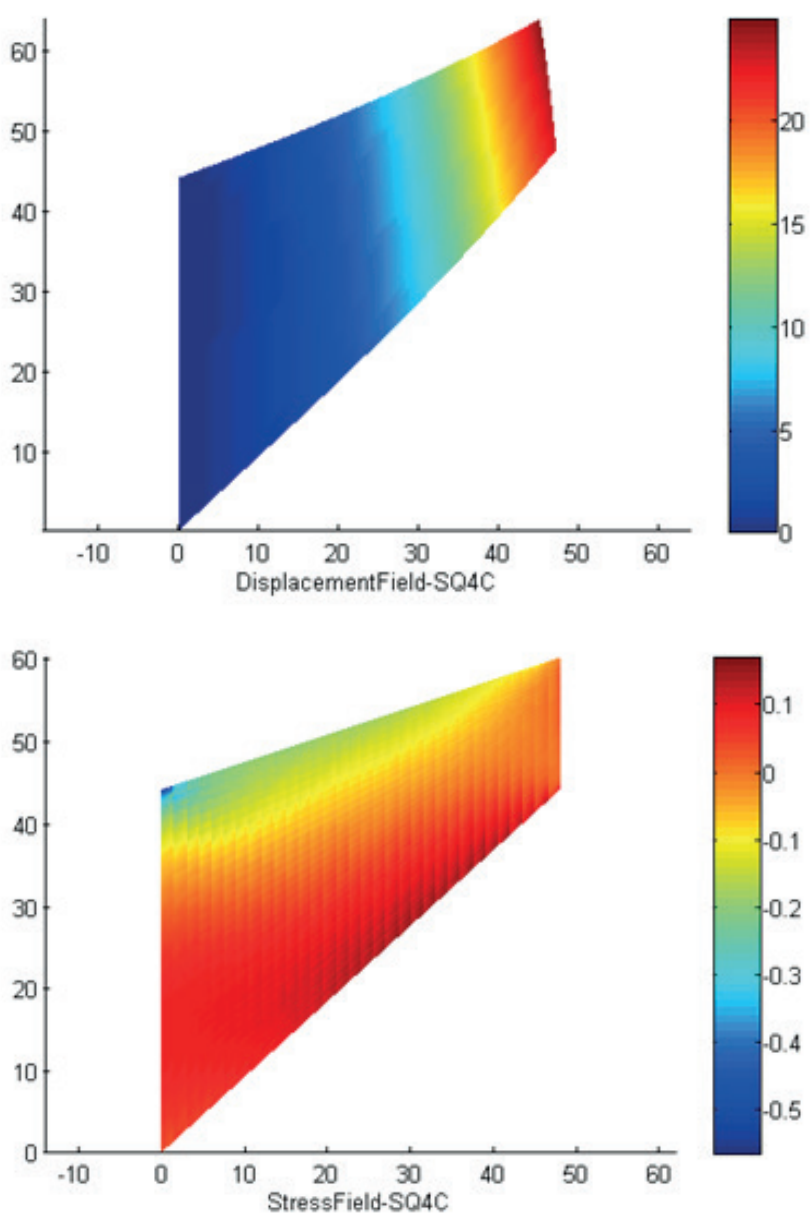

Fig. 10 The displacement field and stress field related to Cook's problem

different values of fiber orientation angles. The results of this study are compared with the numerical results obtained by MQH3T [4], SQUAD4 [11], RDTMLC [12], RDKQ-L24 [13], MISQ20 [4] and the exact result presented by Whitney [14, 15].

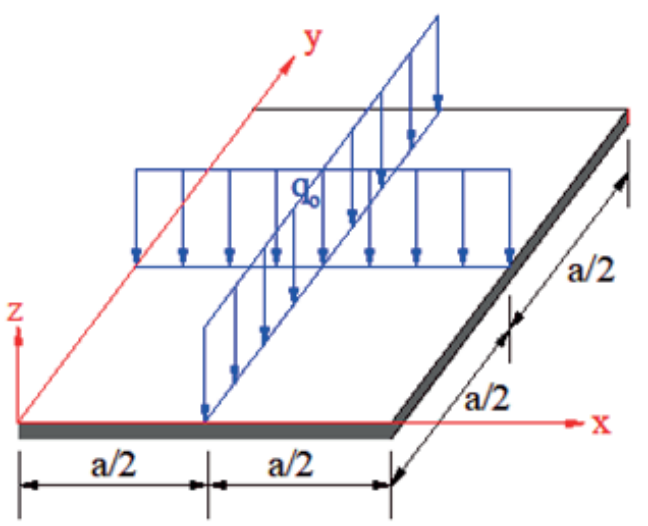

(a)

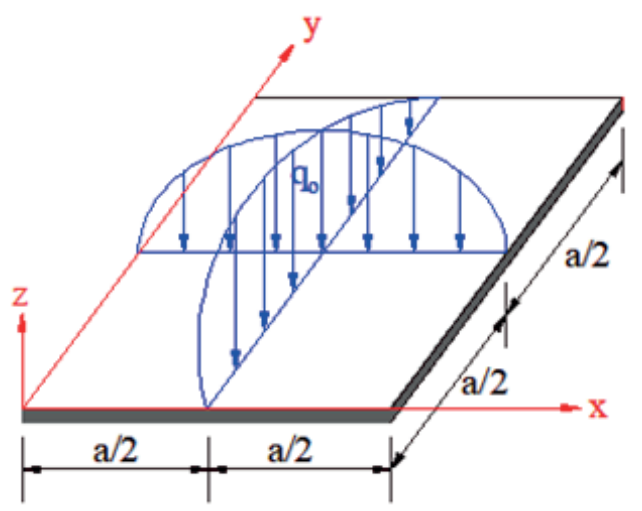

(b)

Fig. 11 The data of geometry with a) uniform load and b) doubly sinusoidal load for unsymmetrical angle-ply square plate

Next, a simply supported two-layer $\left[-45^{\circ} / 45^{\circ}\right]$ with thickness $h$ and length $a$ is subjected to doubly sinusoidal load $q=q_{o} \sin (x / a) \sin (y / a)$ as illustrated in Fig. 11(b). The SCFs are assumed to be $5 / 6$ for this plate made of material $I$. The numerical results for present study are given in Table 5 together with some other results. In this table, the normalized deflection at the plate center $\bar{w}=100 E_{2} w h^{3} /\left(q_{o} a^{4}\right)$, and the

Table 4 The comparison of the normalized central deflections for a clamped square plate with two-layer angle-ply $\left[\theta^{\circ} / \theta^{\circ}\right]$

\begin{tabular}{lccccccc}
\hline$\theta$ & MQH3T & SQUAD4 & RDTMLC & RDKQ-L24 & MISQ20 & SQ4C & Exact \\
\hline $5^{\circ}$ & 0.1083 & 0.1040 & 0.1074 & 0.1049 & 0.1023 & $\mathbf{0 . 1 0 0 0}$ & 0.0946 \\
$15^{\circ}$ & 0.2009 & - & 0.1959 & 0.1993 & 0.1971 & $\mathbf{0 . 1 8 8 4}$ & 0.1691 \\
$25^{\circ}$ & 0.2572 & 0.2602 & 0.2508 & 0.2599 & 0.2580 & $\mathbf{0 . 2 5 0 0}$ & 0.2355 \\
$35^{\circ}$ & 0.2844 & 0.2914 & 0.2782 & 0.2907 & 0.2889 & $\mathbf{0 . 2 8 4 4}$ & 0.2763 \\
$45^{\circ}$ & 0.2929 & 0.3013 & 0.2868 & 0.3004 & 0.2986 & $\mathbf{0 . 2 9 6 0}$ & 0.2890 \\
\hline
\end{tabular}




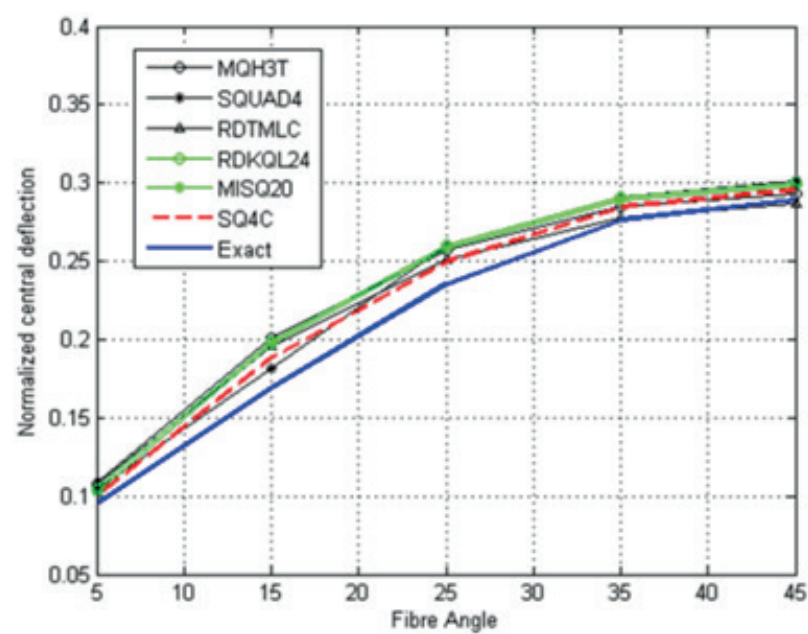

(a)

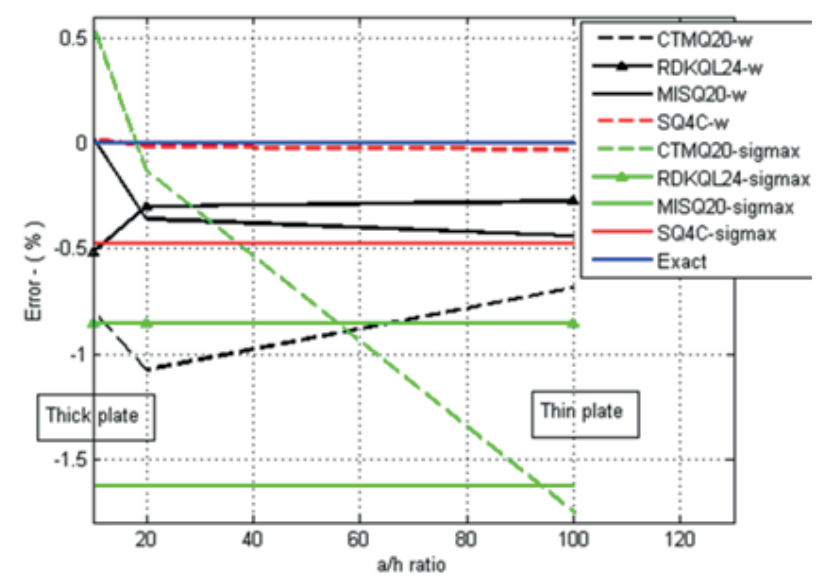

(b)

Fig. 12 a) The convergence of $\bar{w}$ for the case of uniform load and b) The error of $\bar{w}$ and $\overline{\sigma_{x}}$ for case of doubly sinusoidal load

normalized stress $\bar{\sigma}_{x}=\sigma_{x} h^{2} /\left(q_{o} a^{2}\right)$ at point $(a / 2, a / 2, h / 2)$, $\bar{\tau}_{x y}=\tau_{x y} h^{2} /\left(q_{o} a^{2}\right)$ at point $(0,0,-h / 2), \bar{\tau}_{x z}=\tau_{x z} h^{2} /\left(q_{o} a^{2}\right)$ at point $(0, a / 2, h / 4)$ are presented. As compared with the exact solution [7], the errors of the normalized central deflection $\bar{w}$ and the normalized stress $\bar{\sigma}_{x}(a / 2, a / 2, h / 2)$ provided by different types of elements are also shown in Fig. 12(b). The results gained by the SQ4C element are in good agreement with the exact results based on the FSDT from thin to thick plate for arbitrary values $a / h$.

We consider a pinched cylinder of the radius $R=300$ and the length $L=600$ as shown in Fig. 13. This structure is restricted by rigid diaphragms at two ends. At the middle of the length $L$, two opposite radial concentrated loads $P=1$ are applied.

The laminated composite material II with lay-up $\left[0^{\circ} / 90^{\circ}\right],\left[0^{\circ} / 90^{\circ}\right] 5,\left[-45^{\circ} / 45^{\circ}\right]$ or $\left[-45^{\circ} / 45^{\circ}\right]_{5}$ is used for this structure. The different radius-to-thickness ratios $S=R / h$ are investigated.
Table 5 The comparison of normalized central deflection and normalized stresses for a simply supported square plate with two-layer angle-ply $\left[-45^{\circ} / 45^{\circ}\right]$

\begin{tabular}{|c|c|c|c|c|c|}
\hline$a / h$ & Model & $\bar{w}$ & $\overline{\sigma_{x}}$ & $\overline{\tau_{x y}}$ & $\bar{\tau}_{x z}$ \\
\hline \multirow{6}{*}{100} & CTMQ20 [4] & 0.6519 & 0.2474 & 0.2295 & 0.1194 \\
\hline & RDKQ-L24[13] & 0.6546 & 0.2500 & 0.2316 & 0.1597 \\
\hline & MFE [4] & 0.6558 & - & - & - \\
\hline & MISQ20 [4] & 0.6553 & 0.2459 & 0.2304 & 0.1884 \\
\hline & SQ4C & 0.6562 & 0.2486 & 0.2327 & 0.1884 \\
\hline & Exact(FSDT)[7] & 0.6564 & 0.2498 & 0.2336 & 0.2143 \\
\hline \multirow{6}{*}{20} & CTMQ20 [4] & 0.6906 & 0.2523 & 0.2333 & 0.1773 \\
\hline & RDKQ-L24[13] & 0.6960 & 0.2516 & 0.2316 & 0.2020 \\
\hline & MFE [4] & - & - & - & - \\
\hline & MISQ20 [4] & 0.6973 & 0.2456 & 0.2304 & 0.1884 \\
\hline & SQ4C & 0.6980 & 0.2486 & 0.2327 & 0.1884 \\
\hline & Exact(FSDT)[7] & 0.6981 & 0.2498 & 0.2336 & 0.2143 \\
\hline \multirow{6}{*}{10} & CTMQ20 [4] & 0.8218 & 0.2543 & 0.2349 & 0.2005 \\
\hline & RDKQ-L24[13] & 0.8241 & 0.2517 & 0.2316 & 0.2053 \\
\hline & MFE [4] & 0.8257 & - & - & - \\
\hline & MISQ20 [4] & 0.8286 & 0.2459 & 0.2304 & 0.1884 \\
\hline & SQ4C & 0.8286 & 0.2486 & 0.2327 & 0.1884 \\
\hline & Exact(FSDT)[7] & 0.8284 & 0.2498 & 0.2336 & 0.2143 \\
\hline
\end{tabular}

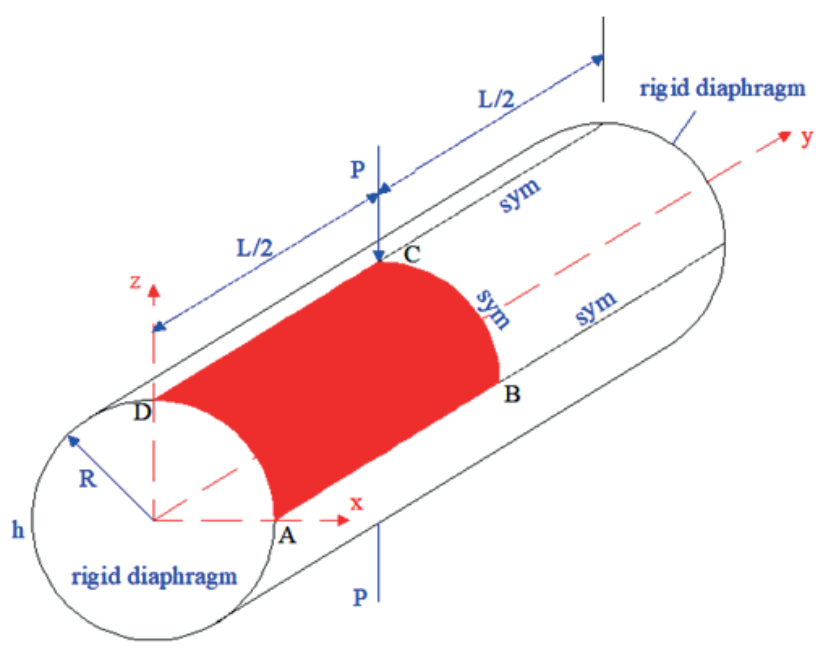

Fig. 13 The rigid diaphragms at two ends of a pinched cylinder

Because of the symmetry of this cylinder, only one octant is modeled with meshes of $32 \times 32$ regular or irregular elements as illustrated in Fig. 14. The normalized displacements at point $C, \bar{w}_{C}=10 E_{1} w_{C} h^{3} /\left(P R^{2}\right)$ are shown in Table 6 with the radius-to-thickness ratios $S=20,50$ and 100 . The SQ4C results for this example are in good agreement with the FSDT results of Reddy [7] and better than results of MISQ20 of Nguyen-Van [4]. 

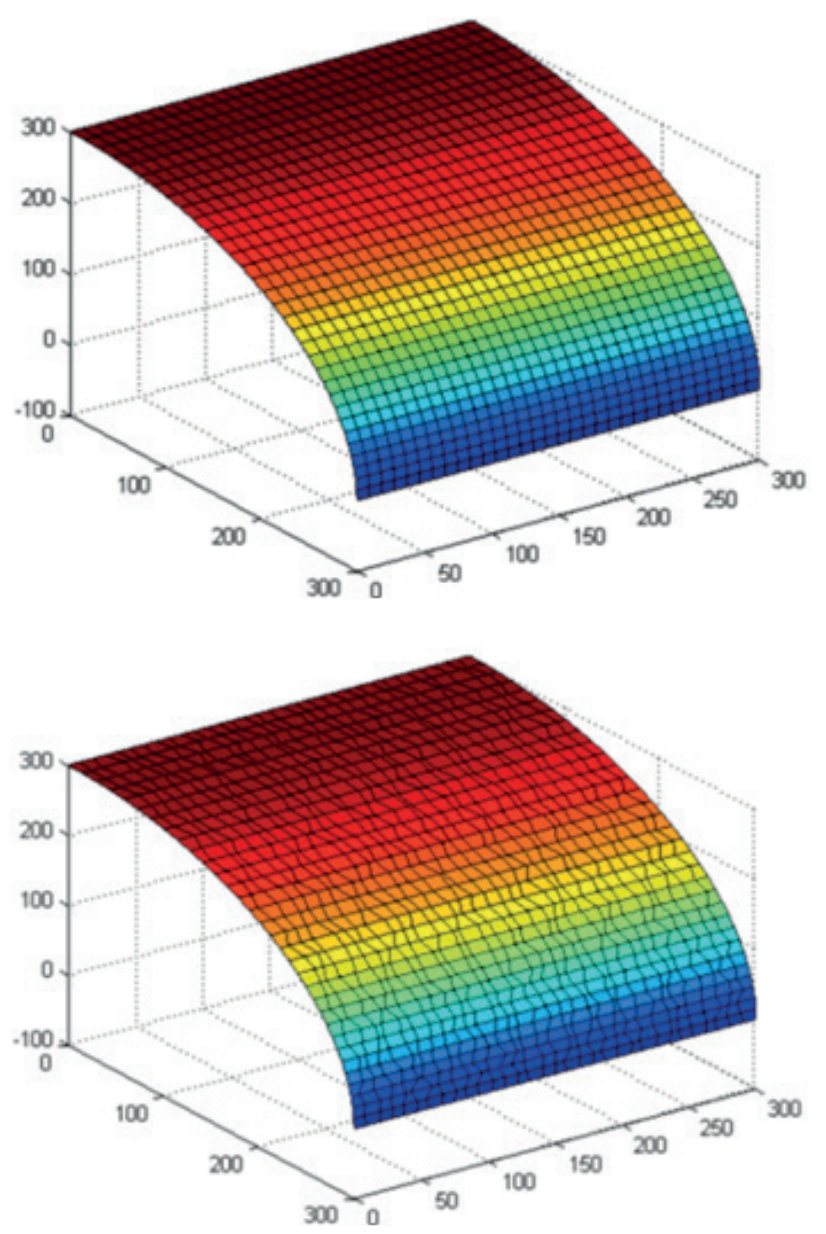

Fig. 14 Two cases with the mesh for one octant of a pinched cylinder

\begin{tabular}{cccccc}
\multicolumn{6}{c}{ Table 6 The comparison of $\bar{w}_{c}$ at point $C$} \\
\hline \multirow{2}{*}{$S=R / h \quad$ Model } & \multicolumn{4}{c}{ Lay-up } \\
& & {$\left[0^{\circ} / 90^{\circ}\right]$} & {$\left[-45^{\circ} / 45^{\circ}\right]$} & {$\left[0^{\circ} / 90^{\circ}\right] 5$} & {$\left[-45^{\circ} / 45^{\circ}\right] 5$} \\
\hline \multirow{4}{*}{20} & MISQ20 [4] & 4.4678 & 5.4868 & 3.7960 & 4.0132 \\
& SQ4C (re) & $\mathbf{4 . 5 2 9 7}$ & $\mathbf{5 . 2 9 2 3}$ & $\mathbf{4 . 1 6 9 8}$ & $\mathbf{3 . 7 5 1 9}$ \\
& SQ4C (irre) & $\mathbf{4 . 6 4 0 0}$ & $\mathbf{5 . 3 9 1 3}$ & $\mathbf{4 . 2 5 6 5}$ & $\mathbf{3 . 7 8 5 4}$ \\
& Reddy [7] & 6.0742 & 5.2275 & 4.2118 & 3.6457 \\
& MISQ20 [4] & 1.7988 & 2.1124 & 1.6559 & 1.3496 \\
50 & SQ4C (re) & $\mathbf{1 . 8 7 3 4}$ & $\mathbf{2 . 1 5 0 5}$ & $\mathbf{1 . 5 5 3 1}$ & $\mathbf{1 . 3 3 4 9}$ \\
& SQ4C (irre) & $\mathbf{1 . 9 4 6 2}$ & $\mathbf{2 . 2 5 6 1}$ & $\mathbf{1 . 6 0 4 4}$ & $\mathbf{1 . 3 2 0 0}$ \\
& Reddy [7] & 2.3756 & 2.2283 & 1.4527 & 1.2986 \\
& MISQ20 [4] & 0.8162 & 1.0500 & 0.4895 & 0.6238 \\
& SQ4C (re) & $\mathbf{0 . 9 4 3 3}$ & $\mathbf{1 . 0 9 9 1}$ & $\mathbf{0 . 5 3 8 2}$ & $\mathbf{0 . 6 8 5 3}$ \\
& SQ4C (irre) & $\mathbf{1 . 0 6 7 5}$ & $\mathbf{1 . 1 1 7 0}$ & $\mathbf{0 . 5 5 0 6}$ & $\mathbf{0 . 6 9 2 5}$ \\
& Reddy [7] & 1.2450 & 1.3065 & 0.7405 & 0.7373 \\
\hline
\end{tabular}

The last example in this section is a shallow spherical shell of the radius $R=10$ and $a=1$ as shown in Fig. 15 . This structure is simply supported on four curved sides as well as subjected to uniform load $q=1$. This shallow shell is manufactured by nine-layer cross-ply $\left[\left(0^{\circ} / 90^{\circ}\right)_{4} / 0^{\circ}\right]$ or angle-ply $\left[\left(45^{\circ} /-45^{\circ}\right)_{4} / 45^{\circ}\right]$ laminates of material $I$ with $E_{1} / E_{2}=40$. The span-to-thickness ratios $a / h=100$ or 1000 are considered.

Based on the symmetry of structure, only one quadrant is simulated as in Fig. 15. The vertical deflections $w_{A}$ at the center point $A$ of the shell with several span-to-thickness ratios $a / h$ determined by the SQ4C elements are compared with other references in Table 7. Table 7 shows the accuracyof the present method with very favorable references $[4,16-19]$.

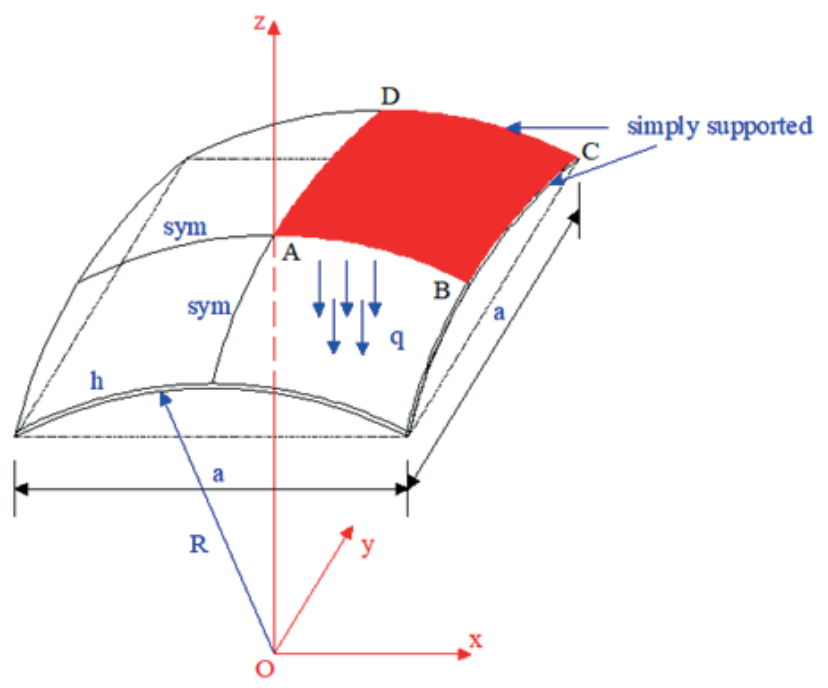

Fig. 15 A shallow spherical shell

Table 7 The central deflection $w_{A} \times 10^{-3}$ with several ratios $a / h$ for the laminated composite shallow spherical shells

\begin{tabular}{|c|c|c|c|}
\hline \multirow[t]{2}{*}{$a / h$} & \multirow{2}{*}{ Model } & \multicolumn{2}{|c|}{ Lay-up } \\
\hline & & {$\left[\left(0^{\circ} / 90^{\circ}\right) 4 / 0^{\circ}\right]$} & {$\left[\left(45^{\circ} /-45^{\circ}\right) 4 / 45^{\circ}\right]$} \\
\hline \multirow{6}{*}{100} & To and Wang [16] & 2.7170 & 0.5259 \\
\hline & Park et al. [17] & 2.7010 & 0.5337 \\
\hline & Somashekar et al. [18] & 2.7270 & 0.5270 \\
\hline & MISQ20 [4] & 2.8005 & 0.5369 \\
\hline & SQ4C & 2.8421 & 0.5399 \\
\hline & Analytic [19] & 2.7170 & 0.5170 \\
\hline \multirow{6}{*}{1000} & To and Wang [16] & 0.0588 & 0.0101 \\
\hline & Park et al. [17] & 0.0591 & 0.0105 \\
\hline & Somashekar et al. [18] & 0.0599 & 0.0088 \\
\hline & MISQ20 [4] & 0.0592 & 0.0063 \\
\hline & SQ4C & 0.0575 & 0.0088 \\
\hline & Analytic [19] & 0.0592 & 0.0105 \\
\hline
\end{tabular}




\subsection{Free vibration analysis}

A simply supported four-layer cross-ply $\left[0 \% 90 \% 90^{\circ} / 0^{\circ}\right]$ square laminated plate made of material I is used to study the frequency convergence of the present elements. With the length-to-thickness ratio $a / h=5$ and a variety of the ratios $E_{1} / E_{2}$, the normalized fundamental frequencies $\bar{\omega}=\left(\omega L^{2} / h\right) \sqrt{\rho / E_{2}}$ related to the SQ4C elements are compared with other results in Table 8 and Fig. 16(a). The

Table 8 The convergence of $\bar{\omega}$ for a simply supported $\left[0 \% 90 \% / 90 \% 0^{\circ}\right]$ square plate with the length-to-thickness ratio $a / h=5$

\begin{tabular}{lcccc}
\hline \multirow{2}{*}{ Model } & \multicolumn{4}{c}{$E_{1} / E_{2}$} \\
& 10 & 20 & 30 & 40 \\
\hline MISQ20 [4] & 8.309 & 9.569 & 10.322 & 10.847 \\
MLSDQ [20] & 8.292 & 9.561 & 10.320 & 10.849 \\
RBF [21] & 8.310 & 9.580 & 10.349 & 10.864 \\
SQ4C & $\mathbf{8 . 3 0 8}$ & $\mathbf{9 . 5 6 7}$ & $\mathbf{1 0 . 3 2 0}$ & $\mathbf{1 0 . 8 4 3}$ \\
Exact & 8.298 & 9.567 & 10.326 & 10.854 \\
\hline
\end{tabular}

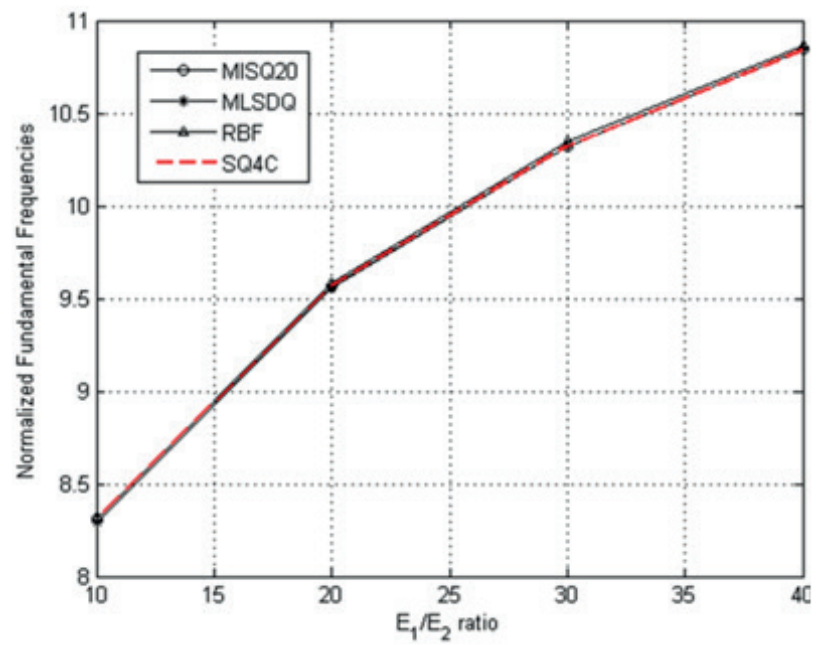

(a)

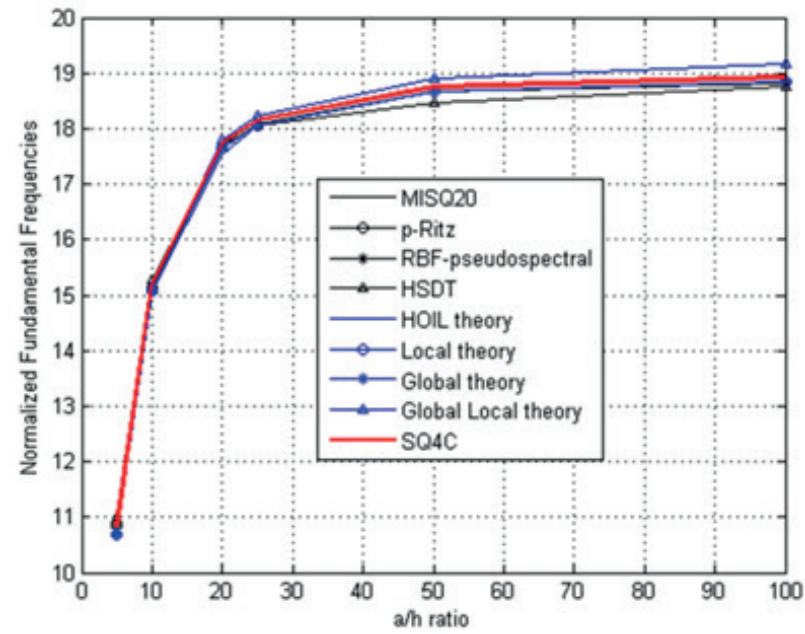

(b)

Fig. 16 Convergence of the $\bar{\omega}$ with a) several $E_{1} / E_{2}$ ratios and b) several a/h ratios

present results based on the SQ4C elements are in good agreement with MLSDQ's results by Liew et al. [20], RBF's results of Ferreira et al. [21], MISQ20's results of NguyenVan [4] and exact results in [7, 22]. The first six mode shapes are also depicted in Fig. 17. To investigate the effect of the length-to-thickness ratio $a / h$ on the fundamental frequency of structure, the normalized fundamental frequencies given by the SQ4C elements in the cases of $E_{1} / E_{2}=40$ and different ratios $a / h$ are shown in Table 9 and Fig. 16(b).

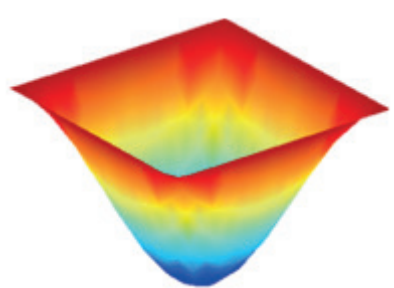

Mode 1

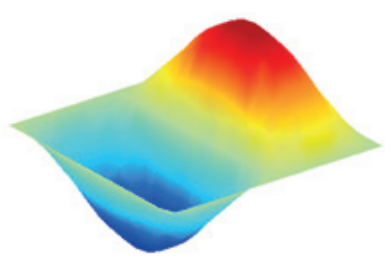

Mode 3

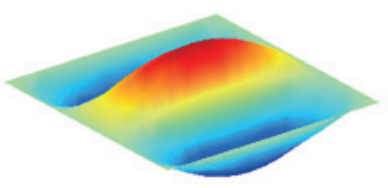

Mode 5

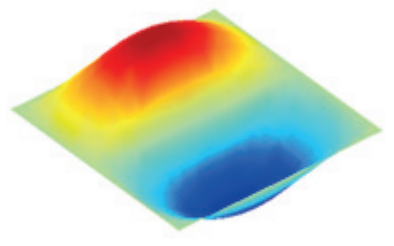

Mode 2

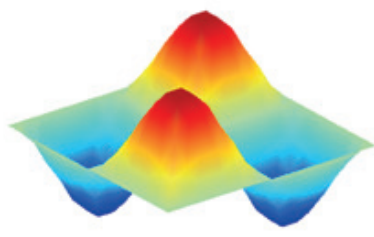

Mode 4

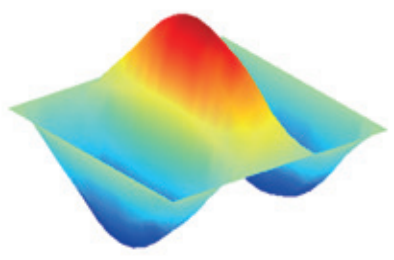

Mode 6

Fig. 17 The first six mode shapes of a simply supported $\left[0 \% 90 \% 90 \% 0^{\circ}\right]$ square plate with $E_{1} / E_{2}=40$ and $a / h=5$

Table 9 Simply supported $\left[0^{\circ} / 90^{\circ} / 90^{\circ} / 0^{\circ}\right]$ square plate with $E_{1} / E_{2}=40$ : Convergence of normalized fundamental frequencies $\bar{\omega}=\left(\omega L^{2} / h\right) \sqrt{\rho / E_{2}}$

\begin{tabular}{lcccccc}
\hline & \multicolumn{6}{c}{$a / h$} \\
Model & 5 & 10 & 20 & 25 & 50 & 100 \\
\hline MISQ20 & 10.847 & 15.165 & 17.719 & 18.138 & 18.753 & 18.918 \\
p-Ritz & 10.855 & 15.143 & 17.658 & 18.071 & 18.673 & 18.836 \\
$\begin{array}{l}\text { RBF-pseudo- } \\
\text { spectral }\end{array}$ & 10.807 & 15.100 & 17.633 & 18.049 & 18.658 & 18.822 \\
$\begin{array}{l}\text { HSDT } \\
\text { HOIL theory }\end{array}$ & 10.989 & 15.268 & 17.666 & 18.049 & 18.462 & 18.756 \\
Local theory & 10.682 & 15.069 & 17.636 & 18.055 & 18.670 & 18.835 \\
Global theory & 10.687 & 15.072 & 17.636 & 18.055 & 18.670 & 18.835 \\
$\begin{array}{l}\text { Global-local } \\
\text { theory }\end{array}$ & 10.729 & 15.165 & 17.803 & 18.240 & 18.902 & 19.156 \\
SQ4C & 10.843 & 15.190 & 17.732 & 18.147 & 18.756 & 18.919 \\
\hline
\end{tabular}


The present numerical results are comparable with solutions of HSDT [23], p-Ritz method [24], RBF-pseudospectral method [25], local higher-order theory [26], global higher-order theory [27], HOIL theory [28], global-local higher-order theory [29] and FSDT-MISQ20 elements [4].

Next, the simply supported cross-ply $\left[0^{\circ} / 90^{\circ} / 90^{\circ} / 0^{\circ}\right]$ laminated cylindrical panel with geometrical properties $L=20, R=100$ and $\varphi=0.1$ radian is given. The thickness $h$ of this structure is 0.2. Each layer has same thickness as well as material II with others.

Due to symmetry, a quadrant nominated as $\mathrm{ABCD}$ as depicted in Fig. 18 is calculated.

The results of the normalized fundamental frequencies given by the SQ4C and references are presented in Table 10. The fundamental frequency provided by the suggested elements are also similar to other numerical results of Nguyen-Van [4] using MISQ20 elements, Liu and To [30] based on layer-wise shell elements, Jayasankar et al. [31] following 9-node degenerated shell elements and the analytical result of Reddy [19]. The first six mode shapes of quadrant ABCD are illustrated in Fig. 19.

A clamped nine-layered cross-ply $[0 \% 90 \% 0 \% 90 \% \%$ $90^{\circ} / 0^{\circ} / 90^{\circ} / 0^{\circ}$ ] laminated spherical panel as presented in Fig. 20 is studied. This structure has side length $a=1$,

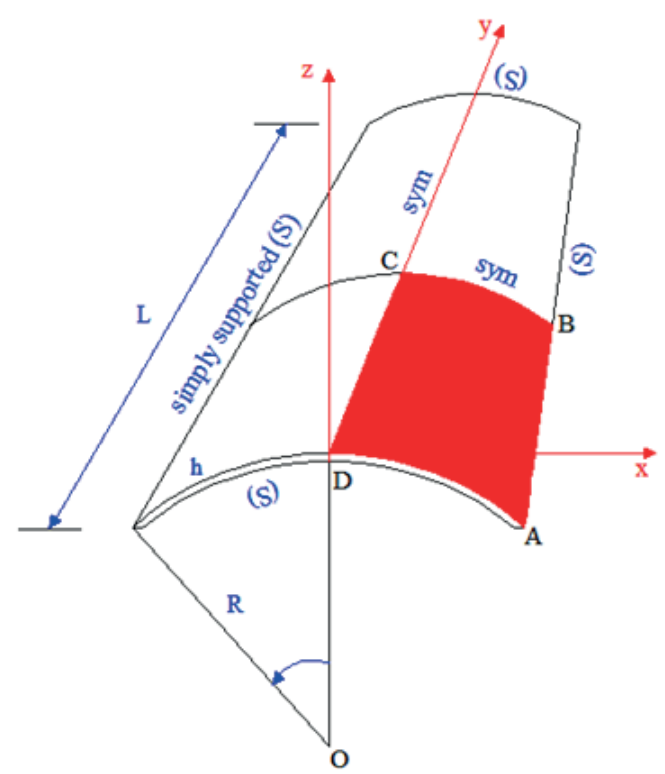

Fig. 18 The data of a laminated cylindrical panel

Table 10 Simply supported cross-ply $\left[0^{\circ} / 90^{\circ} / 90^{\circ} / 0^{\circ}\right]$ cylindrical panel: The $\bar{\omega}=\left(\omega L^{2} / h\right) \sqrt{\rho / E_{2}}$

\begin{tabular}{lccccc}
\hline Lay-up & \multicolumn{5}{c}{ Model } \\
\hline$\left[0^{\circ} / 90^{\circ} / 90^{\circ} / 0^{\circ}\right]$ & MISQ20 & LW theory & Jayasankar & Reddy & SQ4C \\
& {$[4]$} & {$[30]$} & {$[31]$} & {$[19]$} & \\
& 16.736 & 17.390 & 17.700 & 16.668 & $\mathbf{1 6 . 6 5 9}$ \\
\hline
\end{tabular}

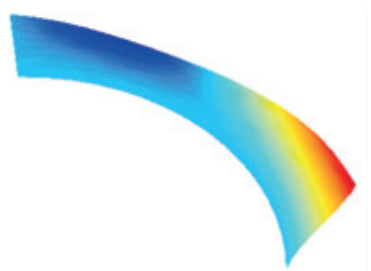

Mode 1

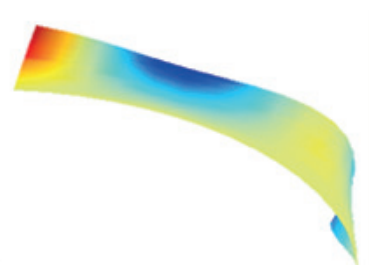

Mode 3

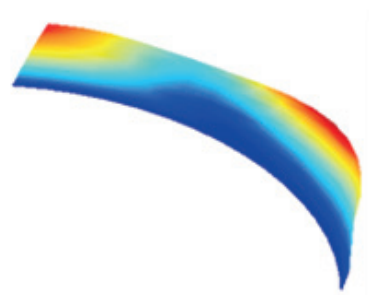

Mode 5

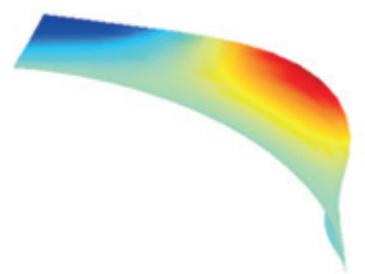

Mode 2

Mode 4

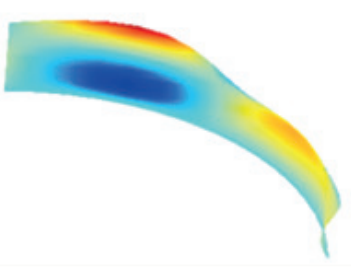

Mode 6

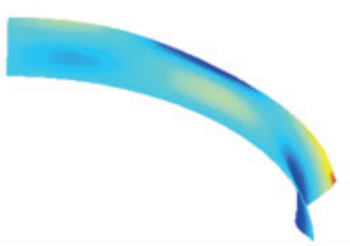

Fig. 19 The first six modes shapes for $A B C D$ part with $\left[0 \% 90 \% / 90 \% 0^{\circ}\right]$ cylindrical panel
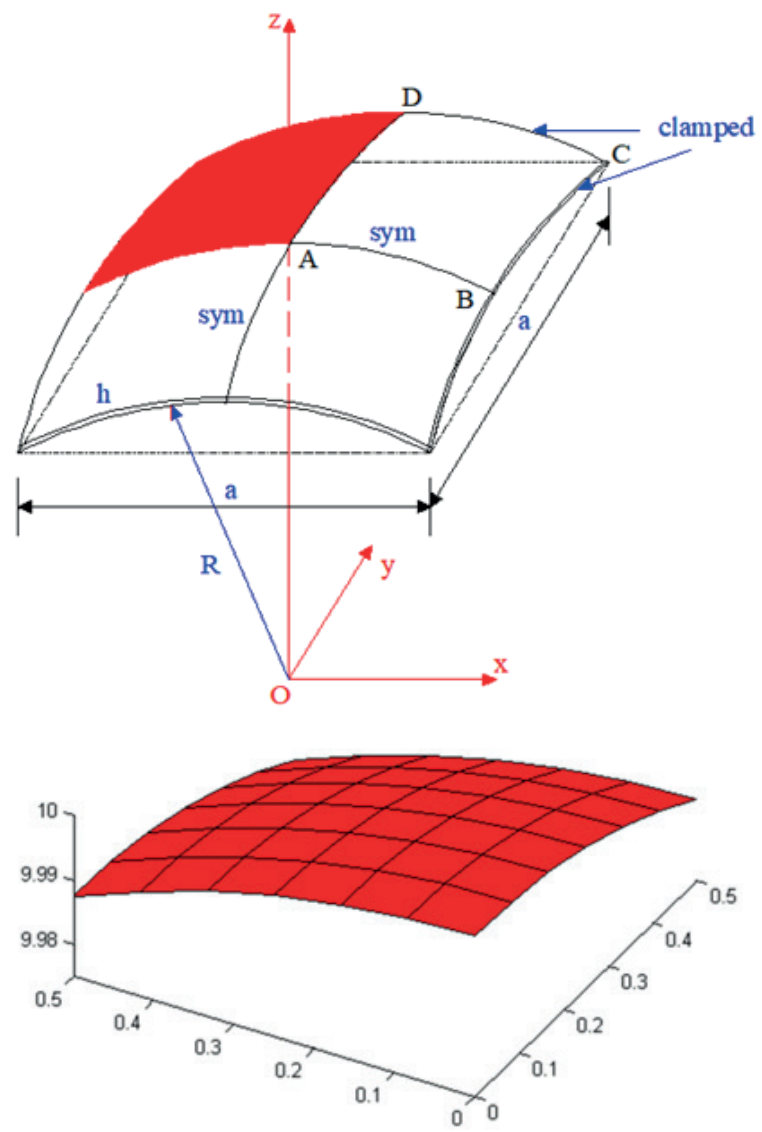

Fig. 20 The laminated spherical panel with mesh for a quadrant 
thickness $h=0.01$ and radius $R=10$. All layers are of same thickness as well as same material III. The mesh of $6 \times 6$ four-node quadrilateral elements is used for modeling a quadrant of shell as shown in Fig. 20.

Table 11 presents the first four normalized natural frequencies related to the SQ4C elements and they are compared with the solutions of Jayasankar et al. [31] using nine-node degenerated shell elements and results of MISQ20 elements using smoothed finite element method by Nguyen-Van [4]. It can be seen that the results obtained by SQ4C elements agree well with the solutions given by Jayasankar et al. [31] and Nguyen-Van [4]. The first six mode shapes are illustrated in Fig. 21.

\subsection{Buckling analysis}

A simply supported four-layer cross-ply $\left[0 \% 90^{\circ} / 90^{\circ} / 0^{\circ}\right]$ square laminated plate of the span $a$ and the thickness $h$ is subjected to uniaxial compression as shown in Fig. 22. The span-to-thickness ratio $a / h$ is 10 . This structure is made of material I with different values of the ratios $E_{1} / E_{2}$.

Table 12 and Fig. 23(a) show that the normalized critical buckling loads of the present element are in good agreement with other results in the cases of different ratios $E_{1} / E_{2}$.

Next, we study the effect of the ratio $a / h$ on the uniaxial critical buckling load for this structure made of material $I$ having $E_{1} / E_{2}=40$. The results achieved by the SQ4C ele-ments are listed in Table 13 and Fig. 23(b). These results also agree well with others achieved by FSDT(a) of Chakrabarti and Sheikh [35], FSDT(b) of Reddy and Phan [23], MISQ20 of Nguyen-Van [4] and HSDT of Reddy and Phan [23].

The uniaxial buckling analysis of simply supported fivelayer $\left[0^{\circ} / 90^{\circ} / 0^{\circ} / 90^{\circ} / 0^{\circ}\right]$ cylindrical shell panel of material $I$ is finally studied as depicted in Fig. 24. The panel is simply supported at all the edges and has ratio $a / b=1$ and $R / a=20$. The SCFs $k_{1}^{2}=k_{2}^{2}=\pi^{2} / 12$ are used for this computation. Table 14 presents the normalized critical buckling loads achieved by SQ4C elements in several cases of $a / h$. In comparison with the solutions of the smoothed

Table 11 Clamped nine-layer $\left[\left(0^{\circ} / 90^{\circ}\right)_{4} / 0^{\circ}\right]$ cross-ply spherical panel: the normalized frequencies

\begin{tabular}{lcccc}
\hline Model & Mode 1 & Mode 2 & Mode 3 & Mode 4 \\
\hline Jayasankar et al. [31] & 67.43 & 84.16 & 99.71 & 113.70 \\
MISQ20 [4] & 67.51 & 86.00 & 101.27 & 115.88 \\
SQ4C & $\mathbf{6 7 . 7 9}$ & $\mathbf{8 5 . 2 1}$ & $\mathbf{1 0 0 . 8 9}$ & $\mathbf{1 1 6 . 0 4}$ \\
\hline
\end{tabular}

elements [4], the higher order elements [36], and the analytic solution [37], the elements SQ4C display the same results. It is sighted that increasing $a / h$ leads to higher value of critical buckling loads.

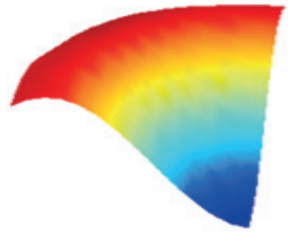

Mode 1

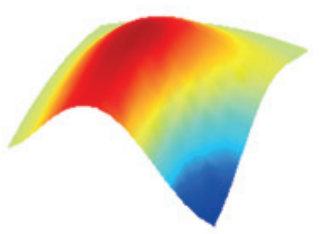

Mode 3

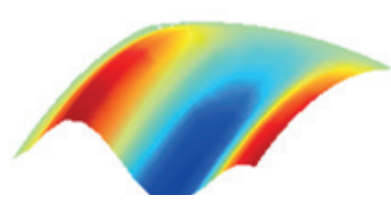

Mode 5

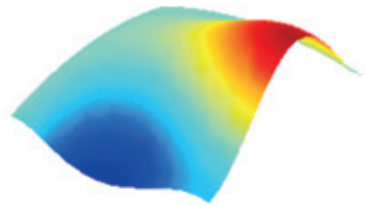

Mode 2

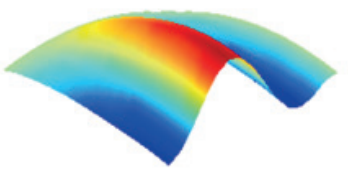

Mode 4

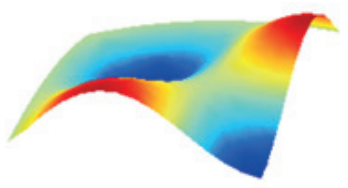

Mode 6
Fig. 21 Clamped nine-layer cross-ply $\left[\left(0^{\circ} / 90^{\circ}\right)_{4} / 0^{\circ}\right]$ spherical panel: the first six mode shapes
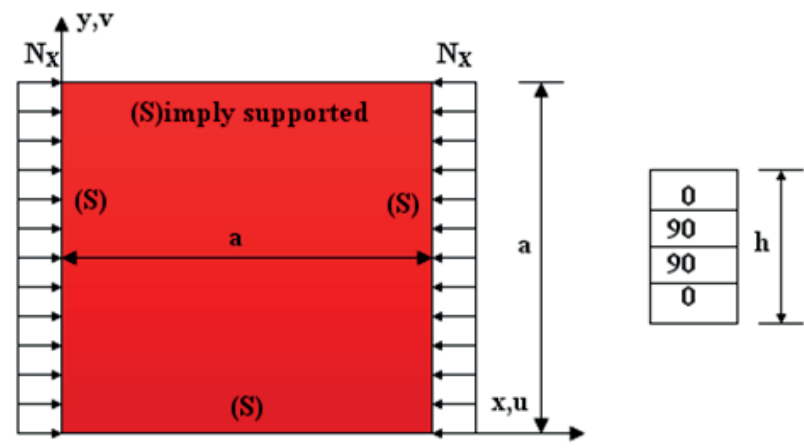

Fig. 22 Simply supported cross-ply $\left[0^{\circ} / 90^{\circ} / 90^{\circ} / 0^{\circ}\right]$ square plate under in-plane uniaxial compression

Table 12 Simply supported cross-ply $\left[0 \% / 90 \% / 90^{\circ} / 0^{\circ}\right]$ square plate: Normalized critical buckling loads $\lambda^{*}=N_{x} a^{2} /\left(E_{2} h^{3}\right)$ with various $E_{1} / E_{2}$ ratios

\begin{tabular}{lccccc}
\hline \multirow{2}{*}{ Model } & \multicolumn{5}{c}{$E_{1} / E_{2}$} \\
& 3 & 10 & 20 & 30 & 40 \\
\hline MISQ20[4] & 5.352 & 9.878 & 15.214 & 19.577 & 23.236 \\
Liu et al. [32] & 5.401 & 9.985 & 15.374 & 19.537 & 23.154 \\
Phan and Reddy [33] & 5.114 & 9.774 & 15.298 & 19.957 & 23.340 \\
Khdeir [22] & 5.442 & 10.026 & 15.418 & 19.813 & 23.489 \\
Noor [34] & 5.294 & 9.762 & 15.019 & 19.304 & 22.881 \\
SQ4C & $\mathbf{5 . 3 5 7}$ & $\mathbf{9 . 8 9 9}$ & $\mathbf{1 5 . 2 6 8}$ & $\mathbf{1 9 . 6 6 8}$ & $\mathbf{2 3 . 3 6 6}$ \\
\hline
\end{tabular}



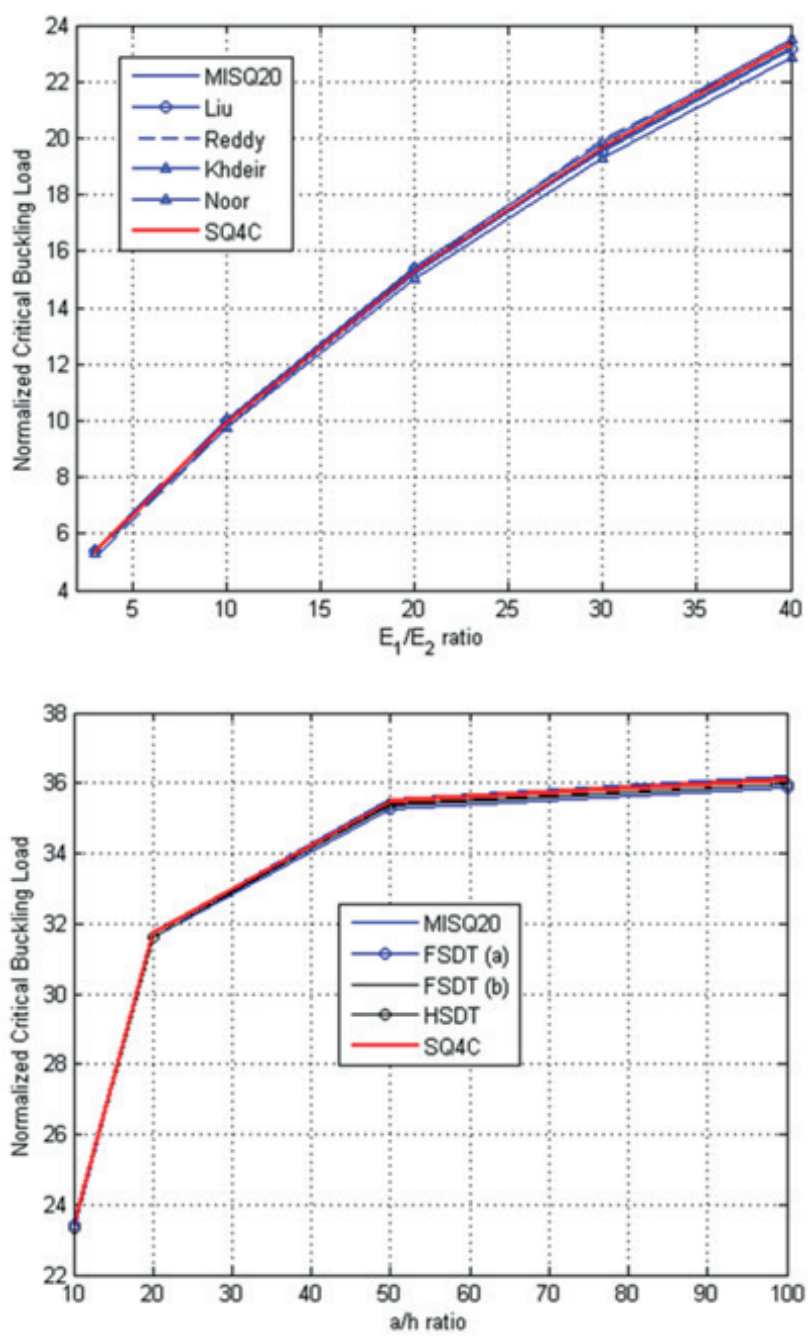

Fig. 23 Normalized critical buckling loads of the simply supported symmetric cross-ply $\left[0 \% 90^{\circ} / 90^{\circ} / 0^{\circ}\right]$ square plate with a) various ratios $E_{1} / E_{2}$ and b) various ratios $a / h$

\section{Conclusions}

In this study, the novel 4-node quadrilateral flat shell element SQ4C is introduced and successfully applied to analysis of laminated composite plate/shell structures in the framework of the FSDT. Numerical analyses of statics, frequency and buckling have been implemented to confirm the robustness and the accuracy of the proposed element. The present element can yield satisfactory results

\section{References}

[1] Yang, H. T. Y., Saigal, S., Masud, A., Kapania, R. K. "A survey of recent shell finite elements", International Journal for Numerical Methods in Engineering, 47(1-3), pp. 101-127, 2000.

https://doi.org/10.1002/(SICI)1097-0207(20000110/30)47:1/3< 101::AID-NME763>3.0.CO;2-C

[2] Ko, Y., Lee, P.-S., Bathe, K.-J. "The MITC4+ shell element and its performance", Computers \& Structures, 169, pp. 57-68, 2016. https://doi.org/10.1016/j.compstruc.2016.03.002
Table 14 Simply supported cross-ply $\left[0^{\circ} / 90^{\circ} / 0^{\circ} / 90^{\circ} / 0^{\circ}\right]$ cylindrical shell panel with

\begin{tabular}{lccccc}
\hline Model & \multicolumn{5}{c}{$a / h$} \\
& 10 & 20 & 30 & 50 & 100 \\
\hline MISQ20 [4] & 23.97 & 31.91 & 34.08 & 35.33 & 35.89 \\
Kumar et al. [36] & 23.97 & 31.79 & - & 35.40 & 36.85 \\
Prusty and & 23.96 & 31.89 & 33.98 & 36.84 & 35.39 \\
Satsangi [38] & & & & & \\
FSDT [37] & 24.19 & 31.91 & 34.04 & 35.42 & 36.86 \\
SQ4C & 24.10 & 31.96 & 34.12 & 35.35 & 35.90 \\
\hline
\end{tabular}

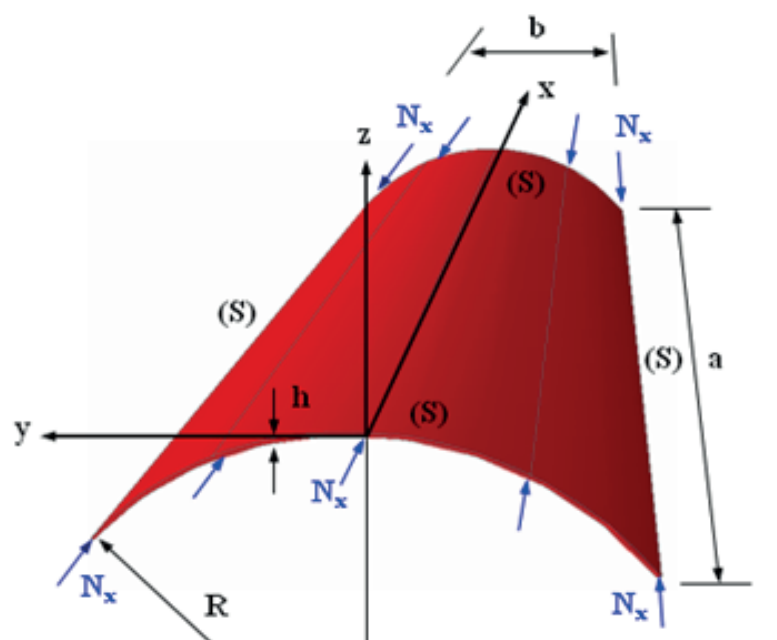

(S)imply supported

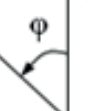

Fig. 24 The data of a cylindrical shell panel

in comparison with other available numerical results. It is also observed that the present approach remains accurate for analysis of both moderately thin and thick plate and shell structures. In addition, the present element has advantages of being simple in formulation and implementation to static, frequency and buckling analyses of both plate and shell structures.

\section{Conflict of interest}

Authors declare that they have no conflicts of interest.

[3] Ton-That, H. L., Nguyen-Van, H., Chau-Dinh, T., Huynh-Van, C. "Enhancement to four-node quadrilateral plate elements by using cell-based smoothed strains and higher-order shear deformation theory for nonlinear analysis of composite structures", Journal of Sandwich Structures \& Materials, 22(7), pp. 2302-2329, 2018. https://doi.org/10.1177/1099636218797982 
[4] Hieu, N.-V. "Development and application of assumed strain smoothing finite element technique for composite plate/shell structures", $\mathrm{PhD}$ Thesis, University of Southern Queensland, 2009. [online] Available at: https://eprints.usq.edu.au/6669/

[5] Ton-That, H. L., Nguyen-Van, H., Chau-Dinh, T. "An Improved Four-Node Element for Analysis of Composite Plate/Shell Structures Based on Twice Interpolation Strategy", International Journal of Computational Methods, 17(6), Article number: 1950020, 2020. https://doi.org/10.1142/S0219876219500208

[6] Thai, C. H., Nguyen-Xuan, H., Nguyen-Thanh, N., Le, T.-H., Nguyen-Thoi, T., Rabczuk, T. "Static, free vibration, and buckling analysis of laminated composite Reissner-Mindlin plates using NURBS-based isogeometric approach", International Journal for Numerical Methods in Engineering, 91(6), pp. 571-603, 2012. https://doi.org/10.1002/nme.4282

[7] Reddy, J. N. "Mechanics of Laminated Composite Plates and Shells, Theory and Analysis", CRC Press, Boca Raton, FL, USA, 2003. https://doi.org/10.1201/b12409

[8] Bathe, K.-J., Dvorkin, E. N. "A formulation of general shell elements - the use of mixed interpolation of tensorial components", International Journal for Numerical Methods in Engineering, 22(3), pp. 697-722, 1986.

https://doi.org/10.1002/nme.1620220312

[9] Macneal, R. H., Harder, R. L. "A proposed standard set of problems to test finite element accuracy", Finite Elements in Analysis and Design, 1(1), pp. 3-20, 1985. https://doi.org/10.1016/0168-874X(85)90003-4

[10] Simo, J. C., Rifai, M. S. "A class of mixed assumed strain methods and the method of incompatible modes", International Journal for Numerical Methods in Engineering, 29(8), pp. 1595-1638, 1990. https://doi.org/10.1002/nme.1620290802

[11] Wilt, T. E., Saleeb, A. F., Chang, T. Y. "A mixed element for laminated plates and shells", Computers \& Structures, 37(4), pp. 597611,1990 .

https://doi.org/10.1016/0045-7949(90)90048-7

[12] Ge, Z., Chen, W. "A refined discrete triangular Mindlin element for laminated composite plates", Structural Engineering and Mechanics, 14(5), pp. 575-593, 2002. https://doi.org/10.12989/sem.2002.14.5.575

[13] Zhang, Y. X., Kim, K. S. "Two simple and efficient displacement-based quadrilateral elements for the analysis of composite laminated plates", International Journal for Numerical Methods in Engineering, 61(11), pp. 1771-1796, 2004. https://doi.org/10.1002/nme.1123

[14] Whitney, J. M. "Bending-Extensional Coupling in Laminated Plates Under Transverse Loading", Journal of Composite Materials, 3(1), pp. 20-28, 1969. https://doi.org/10.1177/002199836900300102

[15] Whitney, J. M. "The Effect of Boundary Conditions on the Response of Laminated Composites", Journal of Composite Materials, 4(2), pp. 192-203, 1970. https://doi.org/10.1177/002199837000400205

[16] To, C. W. S., Wang, B. "Hybrid strain-based three-node flat triangular laminated composite shell elements", Finite Elements in Analysis and Design, 28(3), pp. 177-207, 1998.

https://doi.org/10.1016/S0168-874X(97)00037-1
[17] Park, T., Kim, K., Han, S. "Linear static and dynamic analysis of laminated composite plates and shell using a 4-node quasi-conforming shell element", Composite Part B: Engineering, 37(2-3), pp. 237-248, 2006.

https://doi.org/10.1016/j.compositesb.2005.05.007

[18] Somashekar, B. R., Prathap, G., Babu, C. R. "A field-consistent, four-noded, laminated, anisotropic plate/shell element", Computers \& Structures, 25(3), pp. 345-353, 1987. https://doi.org/10.1016/0045-7949(87)90127-1

[19] Reddy, J. N. "Exact Solutions of Moderately Thick Laminated Shells", ASCE Journal of Engineering Mechanics, 110(5), pp. 794 809, 1984.

https://doi.org/10.1061/(ASCE)0733-9399(1984)110:5(794)

[20] Liew, K. M., Huang, Y. Q., Reddy, J. N. "Vibration analysis of symmetrically laminated plates based on FSDT using the moving least squares differential quadrature method", Computer Methods in Applied Mechanics and Engineering, 192(19), pp. 2203-2222, 2003.

https://doi.org/10.1016/S0045-7825(03)00238-X

[21] Ferreira, A. J. M., Roque, C. M. C., Jorge, R. M. N. "Free vibration analysis of symmetric laminated composite plates by FSDT and radial basis functions", Computer Methods in Applied Mechanics and Engineering, 194(39-41), pp. 4265-4278, 2005. https://doi.org/10.1016/j.cma.2004.11.004

[22] Khdeir, A. A., Librescu, L. "Analysis of symmetric cross-ply laminated elastic plates using a higher-order theory: Part II - Buckling and free vibration", Composite Structures, 9(4), pp. 259-277, 1988. https://doi.org/10.1016/0263-8223(88)90048-7

[23] Reddy, J. N., Phan, N. D. "Stability and vibration of isotropic, orthotropic and laminated plates according to a higher-order shear deformation theory", Journal of Sound and Vibration, 98(2), pp. 157-170, 1985. https://doi.org/10.1016/0022-460X(85)90383-9

[24] Liew, K. M. "Solving the vibration of thick symmetric laminates by Reissner/Mindlin plate theory and the p-RITZ method", Journal of Sound and Vibration, 198(3), pp. 343-360, 1996. https://doi.org/10.1006/jsvi.1996.0574

[25] Ferreira, A. J. M., Fasshauer, G. E. "Analysis of natural frequencies of composite plates by an RBF-pseudospectral method", Composite Structures, 79(2), pp. 202-210, 2007. https://doi.org/10.1016/j.compstruct.2005.12.004

[26] Wu, C.-P., Chen, W.-Y. "Vibration And Stability Of Laminated Plates Based On A Local High Order Plate Theory", Journal of Sound and Vibration, 177(4), pp. 503-520, 1994. https://doi.org/10.1006/jsvi.1994.1448

[27] Matsunaga, H. "Vibration and stability of cross-ply laminated composite plates according to a global higher-order plate theory", Composite Structures, 48(4), pp. 231-244, 2000. https://doi.org/10.1016/S0263-8223(99)00110-5

[28] Cho, K. N., Bert, C. W., Striz, A. G. "Free vibrations of laminated rectangular plates analyzed by higher order individual-layer theory", Journal of Sound and Vibration, 145(3), pp. 429-442, 1991. https://doi.org/10.1016/0022-460X(91)90112-W 
[29] Zhen, W., Wanji, C. "Free vibration of laminated composite and sandwich plates using global-local higher-order theory", Journal of Sound and Vibration, 298(1-2), pp. 333-349, 2006.

https://doi.org/10.1016/j.jsv.2006.05.022

[30] Liu, M. L., To, C. W. S. "Free vibration analysis of laminated composite shell structures using hybrid strain based layerwise finite elements", Finite Elements in Analysis and Design, 40(1), pp. 83-120, 2003. https://doi.org/10.1016/S0168-874X(02)00193-2

[31] Jayasankar, S., Mahesh, S., Narayanan, S., Padmanabhan, C. "Dynamic analysis of layered composite shells using nine node degenerate shell elements", Journal of Sound and Vibration, 299(1-2), pp. 1-11, 2007. https://doi.org/10.1016/j.jsv.2006.06.058

[32] Liu, L., Chua, L. P., Ghista, D. N. "Mesh-free radial basis function method for static, free vibration and buckling analysis of shear deformable composite laminates", Composite Structures, 78(1), pp. 58-69, 2007.

https://doi.org/10.1016/j.compstruct.2005.08.010

[33] Phan, N. D., Reddy, J. N. "Analysis of laminated composite plates using a higher-order shear deformation theory", International Journal for Numerical Methods in Engineering, 21(12), pp. 22012219, 1985.

https://doi.org/10.1002/nme.1620211207
[34] Noor, A. K. "Stability of multilayered composite plates", Fibre Science and Technology, 8(2), pp. 81-89, 1975. https://doi.org/10.1016/0015-0568(75)90005-6

[35] Chakrabarti, A., Sheikh, A. H. "Buckling of Laminated Composite Plates by a New Element Based on Higher Order Shear Deformation Theory", Mechanics of Advanced Materials and Structures, 10(4), pp. 303-317, 2003. https://doi.org/10.1080/10759410306754

[36] Kumar, L. R., Datta, P. K., Prabhakara, D. L. "Tension buckling and dynamic stability behaviour of laminated composite doubly curved panels subjected to partial edge loading", Composite Structures, 60(2), pp. 171-181, 2003.

https://doi.org/10.1016/S0263-8223(02)00314-8

[37] Di Sciuva, M., Carrera, E. "Static buckling of moderately thick, anisotropic, laminated and sandwich cylindrical shell panels", AIAA Journal, 28(10), pp. 1782-1793, 1990.

https://doi.org/10.2514/3.10474

[38] Prusty, B. G., Satsangi, S. K. "Finite element buckling analysis of laminated composite stiffened shells", International Journal of Crashworthiness, 6(4), pp. 471-484, 2001. https://doi.org/10.1533/cras.2001.0191 